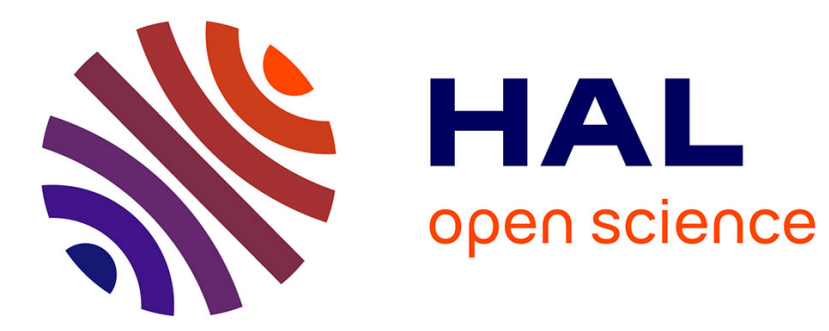

\title{
Nonlinear excitation of subcritical fast ion-driven modes
}

Maxime Lesur, K. Itoh, T. Ido, S.-I. Itoh, Y. Kosuga, M. Sasaki, S. Inagaki,

M. Osakabe, K. Ogawa, A. Shimizu, et al.

\section{To cite this version:}

Maxime Lesur, K. Itoh, T. Ido, S.-I. Itoh, Y. Kosuga, et al.. Nonlinear excitation of subcritical fast ion-driven modes. Nuclear Fusion, 2016, 56 (5), pp.056009. 10.1088/0029-5515/56/5/056009 . hal-02265835

\section{HAL Id: hal-02265835 \\ https://hal.science/hal-02265835}

Submitted on 12 Aug 2019

HAL is a multi-disciplinary open access archive for the deposit and dissemination of scientific research documents, whether they are published or not. The documents may come from teaching and research institutions in France or abroad, or from public or private research centers.
L'archive ouverte pluridisciplinaire HAL, est destinée au dépôt et à la diffusion de documents scientifiques de niveau recherche, publiés ou non, émanant des établissements d'enseignement et de recherche français ou étrangers, des laboratoires publics ou privés. 
Nonlinear excitation of subcritical fast ion-driven modes

This content has been downloaded from IOPscience. Please scroll down to see the full text. 2016 Nucl. Fusion 56056009

(http://iopscience.iop.org/0029-5515/56/5/056009)

View the table of contents for this issue, or go to the journal homepage for more

Download details:

IP Address: 193.50.135.4

This content was downloaded on 12/04/2016 at 15:55

Please note that terms and conditions apply. 


\title{
Nonlinear excitation of subcritical fast ion-driven modes
}

\author{
M. Lesur ${ }^{1,2}$, K. Itoh ${ }^{3,4}$, T. Ido ${ }^{3}$, S.-I. Itoh ${ }^{1,4}$, Y. Kosuga ${ }^{1,5}$, M. Sasaki $^{1}$, \\ S. Inagaki ${ }^{1,3}$, M. Osakabe ${ }^{3,6}$, K. Ogawa $^{3,6}$, A. Shimizu $^{3}$, K. Ida ${ }^{3,6}$ \\ and the LHD experiment group
}

1 Research Institute for Applied Mechanics, Kyushu University, 816-8580 Kasuga, Japan

${ }^{2}$ Institut Jean Lamour, Université de Lorraine, 54506 Vandoeuvre-lès-Nancy Cedex, France

${ }^{3}$ National Institute for Fusion Science, 509-5292 Toki, Japan

${ }^{4}$ Research Center for Plasma Turbulence, Kyushu University, 816-8580 Kasuga, Japan

5 Institute for Advanced Study, Kyushu University, 816-8580 Kasuga, Japan

${ }^{6}$ SOKENDAI (The Graduate University for Advanced Study), 509-5292 Toki, Japan

E-mail: maxime.lesur@polytechnique.org

Received 23 October 2015, revised 7 March 2016

Accepted for publication 10 March 2016

Published 13 April 2016

\begin{abstract}
In collisionless plasma, it is known that linearly stable modes can be destabilized (subcritically) by the presence of structures in phase-space. The growth of such structures is a nonlinear, kinetic mechanism, which provides a channel for free-energy extraction, different from conventional inverse Landau damping. However, such nonlinear growth requires the presence of a seed structure with a relatively large threshold in amplitude. We demonstrate that, in the presence of another, linearly unstable (supercritical) mode, wave-wave coupling can provide a seed, which can lead to subcritical instability by either one of two mechanisms. Both mechanisms hinge on a collaboration between fluid nonlinearity and kinetic nonlinearity. If collisional velocity diffusion is low enough, the seed provided by the supercritical mode overcomes the threshold for nonlinear growth of phase-space structure. Then, the supercritical mode triggers the conventional subcritical instability. If collisional velocity diffusion is too large, the seed is significantly below the threshold, but can still grow by a sustained collaboration between fluid and kinetic nonlinearities. Both of these subcritical instabilities can be triggered, even when the frequency of the supercritical mode is rapidly sweeping. These results were obtained by modeling the subcritical mode kinetically, and the impact of the supercritical mode by simple wave-wave coupling equations. This model is applied to bursty onset of geodesic acoustic modes in an LHD experiment. The model recovers several key features such as relative amplitude, timescales, and phase relations. It suggests that the strongest bursts are subcritical instabilities, with sustained collaboration between fluid and kinetic nonlinearities.
\end{abstract}

Keywords: subcritical instabilities, abrupt excitation, kinetic nonlinearity, geodesic acoustic mode, EGAM, energetic particles

(Some figures may appear in colour only in the online journal)

\section{Introduction}

Subcritical instabilities, which circumvent linear theory, are ubiquitous in fluids and plasmas [1]. These include subcritical or submarginal turbulence, which is self-sustained nonlinearly. Subcritical, or submarginal turbulence, is observed in pipe flow, planar Poiseuille flow, and planar Couette flow, which is linearly stable at all Reynolds numbers [2]. In magnetized fusion plasmas, subcritical turbulence is predicted by theory for current-diffusive interchange turbulence [3], and drift-wave turbulence in slab geometry [4]. Subcritical excitation also concerns large-scale perturbations, such as 
Kelvin-Helmholtz instability $[5,6]$. The formation of selfsustaining magnetic islands (neoclassical tearing mode) is a well-known example in magnetized fusion plasmas [7].

For this wide range of subcritical systems, the subcritical bifurcation originates from a fluid nonlinearity, or nonlinearity in real space. On the other hand, kinetic nonlinearities, or nonlinearities in the phase-space of particle distribution, play a crucial role in hot plasmas. In general, hot plasmas include modes with a wave-length much smaller than the collisional mean-free-path, which enable strong wave-particle resonances. In this regime, particles are free to explore the energy space. This often leads to the formation of structures in the phase-space of particle distribution. This is observed in a wide range of laboratory and space plasmas [8]. The most ubiquitous phase-space structure is a phase-space hole [9], a vortex-like negative phase-space density perturbation, which results from the localized self-consistent trapping of particles by their own electric potential. Phase-space structure formation is a kinetic nonlinearity, in the sense that it cannot be described by fluid models, unlike other nonlinearities such as higher harmonic generation, mode coupling, fluid vortex, etc. Theory predicts that these structures can tap free energy where wave excitation cannot, and lead to subcritical instabilities, where the kinetic nonlinearity enables the growth of a mode that is linearly damped $[10,11]$.

However, such subcritical growth requires a largeamplitude seed perturbation. Several scenarios could provide the seed for kinetic nonlinear growth of a linearly stable mode:

1. the presence of large thermal noise or an external source of wave excitation,

2. a hysteresic path from supercritical to subcritical regime, or

3. a transfer of energy from another, linearly unstable mode.

Previous works on kinetic subcritical instabilities assumed some initial, relatively large amplitude (at least, compared to thermal noise) perturbation [12-14] for the subcritical mode, corresponding to scenario 1 . The hysteretic behavior, corresponding to scenario 2, was obtained in a COBBLEs simulation, and will be the subject of a future paper. Another work explored an artificial scenario, where a seed phase-space hole is imposed at $t=0$ [15].

In [16], a model was developed to explore the third scenario. The model combines the kinetic description of a linearly stable (subcritical) mode with the nonlinear fluid coupling with a prescribed linearly unstable (supercritical) mode. This can be seen as an extension of the Berk-Breizman (BB) model [17] to two interacting modes. The model suggests that the supercritical mode can provide a seed for the nonlinear growth of the subcritical mode. In this paper, we investigate two interesting regimes. In a first regime, of successive fluid then kinetic growth, the dormant subcritical mode is first triggered by fluid coupling to the supercritical mode, which allows it to reach amplitudes of the same order of magnitude as the supercritical mode. This amplitude is above the threshold for the conventional kinetic subcritical instability [12, 14], therefore the amplitude can keep growing by momentum exchange between the wave and phase-space structure(s). In a second regime, of collaborative fluid-kinetic growth, the subcritical growth is due to an uninterrupted collaboration between fluid and kinetic nonlinearities. This is a new kind of instability mechanism, where fluid and kinetic nonlinearities have similar (in amplitude) contributions to the mode growth. Contrarily to the mechanism developed in earlier theories $[12,14]$, the growth occurs much below the amplitude threshold, and without chirping.

As shown in [16], the model qualitatively reproduces an experimental observation, and interprets it as a subcritical instability with essential roles of both fluid and kinetic nonlinearities. In the latter reference, we analyzed an intriguing observation in the helical plasma of the LHD, which was described first in [18]. Bursts of energetic particle-driven geodesic acoustic mode (EGAM) [19], with dynamical evolution of frequency (chirping) are routinely observed, with a $10 \mathrm{~ms}$ duration. Surprisingly, such a primary EGAM burst is sometimes accompanied by a secondary, stronger burst. The secondary burst has a $1 \mathrm{~ms}$ duration, and a peak amplitude that significantly exceeds that of the primary burst. Since the existence of the secondary burst appears to be tied to the primary burst, we call the primary (weaker, and chirping) burst as mother mode, and the secondary burst as daughter mode. The mother EGAM chirps from $50 \mathrm{kHz}$ to $90 \mathrm{kHz}$. When it approaches $80 \mathrm{kHz}$, the daughter mode abruptly appears at $\sim 40 \mathrm{kHz}$, with a growth rate one order of magnitude larger than the mother's. The amplitude increase of the daughter is so large (compared to the amplitude decrease of the mother) that it clearly violates the Manley-Rowe relations [20]. This suggests that the daughter is not excited by e.g. simple parametric coupling. In section 6, we summarize the relevant experimental conditions, and apply our model to a typical daughter burst as shown in figure 5. Our analysis suggests that the daughter mode is a subcritical instability, which is dormant until the mother excites it into the regime of collaborative fluidkinetic growth. This was first reported in [16]. In this paper, we expand on the latter analysis of LHD experiment. We further discuss the applicability of the model to this experiment, and several caveats. We introduce scalar measures to quantify the comparison between experiment and simulation, and use them to analyze the robustness of the modeling with respect to input parameters, as well as to provide readily-testable predictions.

The main point of this paper, though, is to provide more theoretical basis for the reduced model (section 2), explore different regimes (sections 3 and 4), and clarify the underlying physics based on the behavior in simpler limits (section 5).

\section{Model}

The model was introduced in [16]. Here, we expand on required assumptions, their justification or validity range, and what novel physics can be investigated by this model.

\subsection{From $3 D$ to $1 D$}

In a toroidal device, the linear structure, linear frequency and linear growth rate of an energetic particle-driven mode is determined by $3 \mathrm{D}$ calculations. These linear properties 
Table 1. Analogies between a single wave in a 1D plasma, and a single EGAM in a $3 \mathrm{D}$ toroidal plasma.

\begin{tabular}{lll}
\hline & $1 \mathrm{D}$ & $3 \mathrm{D}$ \\
\hline Angle & $k x-\omega t$ & $\theta-\omega t$ \\
Action & $m_{i} \frac{v-v^{R}}{k}$ & $I \sim m_{i} \frac{v_{\|}-v_{\|}^{R}}{k_{\|}}$ \\
Effective mass & $\frac{k^{2}}{m_{i}}$ & $D \approx \frac{k_{\|}^{2}}{m_{i}}$ \\
Electric potential & $\hat{\phi}$ & $\hat{\phi}_{1}$ \\
Electric field & $E$ & $E_{\theta}$ \\
\hline
\end{tabular}

evolve on a slow timescale of mean field evolution ( $100 \mathrm{~ms})$. However, the kinetic nonlinear effects, which induce chirping and subcritical instability, are linked with the evolution on a fast timescale $(\sim 1 \mathrm{~ms})$. They can be treated perturbatively in a $1 \mathrm{D}$ model (the BB model) [21], by taking advantage of the timescale separation. This reduced 1D model is linked to the $3 \mathrm{D}$ mode by a perturbative expansion of a gyrokinetic Hamiltonian around a resonant surface in phase-space [22].

This procedure is applied to an EGAM in appendix. Here, we summarize how wave-particle interactions on a fast timescale may be approximated by a 1D model. Developing the perturbed 3D Hamiltonian $H_{1}$ for an EGAM, in the neighborhood of the flux surface of peak electric potential, yields, as a zeroth-orderin- $\partial H_{0} / \partial J_{\theta}$ approximation, and after substituting the resonance condition $\omega=m \omega_{\theta}$, a $1 \mathrm{D}$ Hamiltonian in a phase-space $(\theta$, $J_{\theta}$ ). Here, $J_{\theta}$ is the canonical poloidal angular momentum. The latter 1D Hamiltonian is isomorphic to the Hamiltonian of a single sine wave in an electrostatic 1D plasma. In this sense, the physics of the 1D model, and that of the reduced 3D model, are analogous. Table 1 summarizes the analogy.

In this work, we consider the interaction of two modes. To treat the present problem, we split the electric field $E$ between the two waves, $E=E_{1}+E_{2}$, and introduce a hybrid model. The subcritical (daughter) mode $\left(E_{1}\right)$ is treated by the kinetic 1D model, and the supercritical (mother) mode $\left(E_{2}\right)$ is treated as a simple medium for nonlinear energy transfer. For $E_{2}$, we prescribe the initial amplitude $Z_{2,0}$ and timeevolution of frequency $\omega_{2}(t)$. We assume that the impact of the mother on the particles near the resonant location of the daughter is negligible. This is a strong assumption, because there is a near-integer frequency ratio at the time of daughter burst. We encourage direct tests of this assumption by first principles. The interaction between the two waves is modeled by the equations for period doubling.

\subsection{Model equations}

We adopt a perturbative approach, and cast the equations for wave-particle interactions in a reduced form, which describes the time evolution of the beam particles only [17]. The main hypothesis in this approach is that the bulk particles interact adiabatically with the wave, so that their contribution to the Lagrangian can be expressed as a part of the electric field. In this model, the linear frequency of the wave $E_{1}$ is fixed. Even when chirping occurs, $\omega_{1}$ does not change. Chirping, when it occurs, is due to the nonlinear evolution of the amplitude and phase of $E_{1}$, rather than the evolution of $\omega_{1}$.

The evolution of the energetic particle distribution, $f(x$, $v, t$, in the neighbourhood of the resonance of the daughter mode $E_{1}$, is given by a kinetic equation $[17,23]$,

$$
\frac{\partial f}{\partial t}+v \frac{\partial f}{\partial x}+\frac{q E_{1}}{m} \frac{\partial f}{\partial v}=\frac{\nu_{f}^{2}}{k_{1}} \frac{\partial \delta f}{\partial v}+\frac{\nu_{d}^{3}}{k_{1}^{2}} \frac{\partial^{2} \delta f}{\partial v^{2}},
$$

where $\delta f \equiv f-f_{0}$, and $f_{0}(v)$ is the initial velocity distribution.

The rhs is a collision operator, where $\nu_{f}$ and $\nu_{d}$ are input parameters characterizing dynamical friction and velocityspace diffusion, respectively. This operator can be obtained by projecting a Fokker-Planck operator [24] that describes Coulomb collisions perceived by energetic ions, on the resonant phase-space surface $[23,25,26]$.

The evolution of the two parts of electric field is given by

$$
\begin{aligned}
\frac{\mathrm{d} Z_{1}}{\mathrm{~d} t}= & -\frac{m \omega_{1}^{3}}{4 \pi q^{2} n_{0}} \int f(x, v, t) \mathrm{e}^{-\mathrm{i}\left(k_{1} x-\omega_{1} t\right)} \mathrm{d} x \mathrm{~d} v \\
& -\gamma_{d} Z_{1}-\imath \frac{V}{\omega_{1}} Z_{2} Z_{1}^{*} \mathrm{e}^{-\imath \theta t}, \\
\frac{\mathrm{d} Z_{2}}{\mathrm{~d} t}= & -l \frac{V}{\omega_{2}} Z_{1}^{2} \mathrm{e}^{\imath \theta t},
\end{aligned}
$$

where $E_{j} \equiv Z_{j} \exp \left[\imath\left(k_{j} x-\omega_{j} t\right)\right]+$ c.c., and $n_{0}$ is the total density.

The model above in the limit of no wave coupling $(V=0)$ was described in details in [17]. The perturbed current is obtained by assuming that energetic particles interact with a mode only if their velocity $v_{i}$ is close enough to the mode's phase velocity $v_{\phi}=\omega_{1} / k_{1}$. Terms of the order of $\left(\omega_{b} / \omega_{1}\right)^{2}\left(v_{i}-v_{\phi}\right) / v_{\phi}$ are neglected. Here, $\omega_{b}=\left(|q| k_{1}\left|Z_{1}\right| / m\right)^{1 / 2}$ is the bounce frequency of particles deeply trapped into the potential well. This is a perturbative description, which does not take into account effects of the time-evolution of the bulk particle velocity distribution (assuming the bulk of particles interact adiabatically with the wave). In addition, this model assumes a constant total number of energetic particles.

The term proportional to $\gamma_{d}$ is an external wave damping, which is a model for all linear dissipative mechanisms of the wave energy to the background plasma [17]. Since the time-scale of fast-particle evolution is much faster than background thermal populations evolution, these two dynamics are decoupled. Hence we can reasonably treat the effects of background damping in this extrinsic way. We further assumed that all background damping mechanisms affect linearly the wave energy. Modeling all background damping mechanisms as an extrinsic, fixed linear damping on the wave is a strong assumption, whose validation requires significant theoretical advances in the understanding of these mechanisms. We must assume that $\gamma_{d}$ depends neither on the wave amplitude, nor on the energetic population. In the case of frequency sweeping, the assumption is clearly violated if the nonlinear modification of frequency is of the order of the linear frequency. In particular, this may be an important issue if a chirping phasespace structure approaches the SAW continuum, where 
damping rate depends largely on the frequency. Therefore, application of this reduced model to energetic particle-driven MHD modes in toroidal plasmas is limited to cases where mode 1 is not, or only weakly, chirping.

We note that in this model, we split the electric field into two parts, and assume that there is one class of particles (distribution $f$ ) which does not interact with one of the two parts of the electric field. We consider a system composed of the two waves and the latter class of particles. In this sense, this model system is an open system. Therefore, the total energy of the system is not expected to be conserved. Furthermore, the term in $\gamma_{d}$ is an extrinsic term of prescribed energy exchange with the 'outside world'.

\subsection{Novelty of the model}

Equations (2) and (3) both include a term that describes energy exchange between mother and daughter. The nonlinear interaction between GAMs (zonal flows) has been studied. The dominant interaction originates either from second-order coupling between vorticity and parallel velocity, as well as vorticity and density [27], or via higher-order modulation mechanisms of background turbulence [28, 29], when the conventional $\boldsymbol{v} \cdot \boldsymbol{\nabla} \boldsymbol{v}$ nonlinearity is not efficient.

In both cases, the coupling takes a standard form, which depends on the coupling constant $V$, and the frequency mismatch $\theta \equiv \omega_{2}(t)-2 \omega_{1}$. This choice is guided by the experimental observation on the LHD [18], where a $\sim 40 \mathrm{kHz}$ daughter mode abruptly grows when the mother mode approaches $\sim 80 \mathrm{kHz}$. This is not accidental because 1 . this ratio of $\sim 2$ is observed in all bursts and in different plasma shots; and 2. the dynamics of period doubling has been demonstrated experimentally in the reference.

In this model, the linear frequency of the mode, $\omega_{1}$ is fixed, but the frequency of $E_{1}$ can evolve nonlinearly due to the timeevolution of $Z_{1}$. In contrast, $\omega_{2}$ is a prescribed function of time, which is a model for the nonlinear chirping of mode 2 .

Equation (3) does not include any dissipative term (no $\gamma_{d}$ ) nor driving term, because we assume a balance between external drive and external damping for simplicity. This assumption is consistent with the experiment we analyze in section 6 , given the timescale separation between evolutions of mother and daughter. Indeed, in the experiment, the timescale of evolution of the mother $(\sim 10 \mathrm{~ms})$ is much slower than this of the daughter $(<1 \mathrm{~ms})$, as long as the daughter's amplitude is less than half that of the mother's.

We extended the semi-Lagrangian kinetic code COBBLES [13] to solve the initial-value model described above. The velocity distribution $f_{0}$ is designed with a constant slope, which is measured by the linear drive $\gamma_{L 0}=\left(\pi \omega_{1}^{3}\right) /\left(2 k_{1}^{2} n_{0}\right) \partial_{v} f_{0}$. Drive and damping are defined so that in the limit $V=\nu_{f}=\nu_{d}=0$, the linear growth rate reduces to $\gamma_{0}=\gamma_{L 0}-\gamma_{d}$. The spatial and velocity grids have 128 and 2048 points, respectively. The simulation time-step width is $0.05 \omega_{1}^{-1}$. To simulate thermal noise, we add to $Z_{1}$ a noise term $Z_{\text {noise }} \mathrm{e}^{i \phi_{r}}$, where $\phi_{r}$ is a phase that is randomized at each time step. This is an important component of the modeling, since in our simulations, mode 1
Table 2. Input parameters of the model.

\begin{tabular}{|c|c|c|c|c|}
\hline Parameter & Description & Value & Range & $\begin{array}{l}\text { Independent } \\
\text { estimation }\end{array}$ \\
\hline$\gamma_{L 0} / \omega_{1}$ & $\begin{array}{l}\text { Linear drive } \\
\text { of daughter }\end{array}$ & 0.03 & $0.01-0.08$ & $\begin{array}{l}0.1 \text { is } \\
\text { supercritical [30] }\end{array}$ \\
\hline$\gamma_{d} / \gamma_{L 0}$ & $\begin{array}{l}\text { L. dissipation } \\
\text { of daughter }\end{array}$ & 1.03 & $1.01-1.7$ & $\begin{array}{l}\gamma_{L} \approx \gamma_{d} \\
\text { hypothesis }\end{array}$ \\
\hline$\nu_{f} / \gamma_{L 0}$ & $\begin{array}{l}\text { Collisional } \\
\text { friction }\end{array}$ & 0.067 & $0.003-0.3$ & $\begin{array}{l}\text { Fokker-Planck, } \\
0.068\end{array}$ \\
\hline$\nu_{d} / \gamma_{L 0}$ & $\begin{array}{l}\text { Collisional } \\
\text { diffusion }\end{array}$ & 0.53 & $0.3-1.5$ & $\begin{array}{l}\text { Fokker-Planck, } \\
0.44\end{array}$ \\
\hline$V Z_{0} / \omega_{1}^{2}$ & $\begin{array}{l}\text { Fluid } \\
\text { coupling } \\
\text { coefficient }\end{array}$ & 50 & $40-80$ & $\sim 10^{-2}-10^{2}[29]$ \\
\hline$Z_{\text {noise }} / Z_{2,0}$ & $\begin{array}{l}\text { Noise } \\
\text { amplitude }\end{array}$ & 0.06 & $10^{-4}-0.3$ & Input from \\
\hline $10^{3} Z_{2,0} / Z_{0}$ & $\begin{array}{l}\text { Initial } \\
\text { mother's } \\
\text { amplitude }\end{array}$ & 1 & $0.6-2.0$ & experimental \\
\hline$\omega_{1}^{-2} \mathrm{~d} \omega_{2} / \mathrm{d} t$ & $\begin{array}{l}\text { Mother's } \\
\text { chirping rate }\end{array}$ & $5 \cdot 10^{-4}$ & $10^{-4}-10^{-3}$ & data \\
\hline
\end{tabular}

Note: Here, $Z_{0}$ is an arbitrary normalizing factor.

is linearly damped and the quasi-resonance condition $|\theta| \ll \omega_{1}$ is only satisfied for a limited period of time. Without the noise, the amplitude of mode 1 would quickly decay to values orders-of-magnitudes below thermal noise, effectively disabling fluid coupling.

The input parameters of the model are summarized in table 2, first and second columns. The third column gives reference values that are used in most simulations, apart from exceptions as mentioned later. The choice of reference values, and the meaning of the fourth and fifth columns, correspond to the LHD experiment, as will be explained in section 6 .

Throughout this paper, the frequency of the mother mode, $\omega_{2}$, is chosen as a linear function of time, $\omega_{2}(t)=1.5 \omega_{1}+$ $\left(\mathrm{d} \omega_{2} / \mathrm{d} t\right) t$. The model is consistent with other kinds of slowlyevolving $\omega_{2}$, but we impose this prescription in order to reduce the number of input parameters. The choice of constant slope can be seen as a first-order approximation based on the timescale separation between the mother and the daughter, since we investigate the abrupt growth of the daughter rather than the slow evolution of the mother. The initial frequency mismatch $\theta(0)=-0.5 \omega_{1}$ is arbitrary, but we have checked that the results do not depend on $\theta(0)$ (we have checked the range $\theta(0) / \omega_{1}=-1.0$ to -0.4$)$.

The extension of this model to three interacting modes is straightforward. However, a strong phase relationship between mode 1 and mode 2 in the experiment [18], suggests that the mechanism of energy exchange can be modeled by 2 -waves coupling, without introducing additional input parameters associated with a 3-waves model. In our simulations, the time-evolution of mode 1 is similar whether we adopt the 2-waves model or 3-waves model. Therefore, for the sake of clarity, we do not discuss the 3 -waves model any further in this paper. 


\section{Fluid and kinetic nonlinearities}

Equation (2) contains two nonlinear terms, which we refer to as kinetic nonlinearity (the term proportional to $\left.\int f \mathrm{e}^{-l\left(k_{1} x-\omega_{1} t\right)}\right)$, and fluid nonlinearity (the term proportional to $V Z_{2} Z_{1}^{*} \mathrm{e}^{-\imath \theta t}$ ). Figure 1 summarizes the main message of this paper. It shows the time evolution of a subcritical mode 1 and a supercritical, chirping mode 2, obtained with the same input parameters, as listed in table 2 (third column), with the following exceptions. The fluid nonlinearity is artificially disabled in the left column of the figure, and the kinetic nonlinearity is disabled in the top row of the figure. This figure shows that the fluid nonlinearity $(b)$, and the kinetic nonlinearity $(c)$, can work in collaboration $(d)$ to drive a subcritical instability to relatively large amplitude. Figure 1(e) illustrates the fixed frequency $\omega_{1}$ and the prescribed time-evolution of $\omega_{2}$.

Let us make clear that the message is not that fluid nonlinearity alone cannot drive a subcritical instability to relatively large amplitude. In fact, figure $1(b)$ includes, as dotted curves, a simulation with increased coupling coefficient, $V=400 \omega_{1}^{2} / Z_{0}$. In this case, mode 1 reaches an amplitude similar to the initial amplitude of mode 2 . However, it does so at the expense of an increased depletion of the energy of mode 1 , and with a qualitatively different evolution, in terms of e.g. the timing of the growth phase. The latter timing, in particular, is crucial to interpret experiments. The message is, rather, that the presence of kinetic nonlinearity, everything else being equal, can significantly enhance the subcritical growth of a mode coupled to a supercritical mode.

\section{Phenomenology}

We are interested in a parameter range where the daughter mode is significantly destabilized, with important contributions from both fluid and kinetic nonlinearities. In this parameter range, we can discriminate two interesting regimes (perhaps non-exhaustively):

(i) In one regime, the daughter growth is first triggered by wave-wave fluid coupling to the mother mode, which allows it to reach amplitudes of the same order of magnitude as the mother. This amplitude is above the threshold for the conventional kinetic subcritical instability, therefore the amplitude can keep growing by momentum exchange between the wave and phase-space structure(s). In this case, the daughter mode chirps significantly, and its amplitude can grow one or more order(s)-of-magnitude above the mother.

(ii) In another regime, the daughter growth is due to an uninterrupted collaboration between fluid and kinetic nonlinearities. This is a new kind of instability mechanism, where fluid and kinetic nonlinearities have similar (in amplitude) contributions to the mode's growth. In this case, the daughter mode is not, or very weakly chirping, and its amplitude stays within the same orderof-magnitude as the mother. In section 6, we interpret an experimental observation of EGAM in the LHD as an example of this subcritical instability.
Without fluid coupling

With fluid coupling
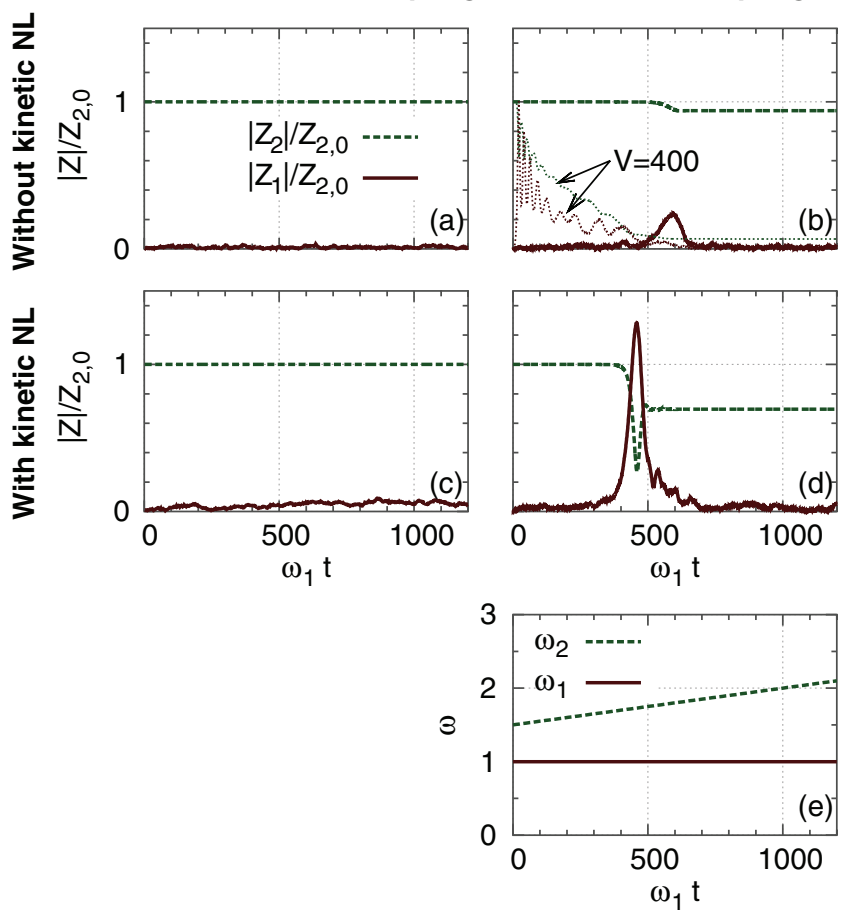

Figure 1. Time evolution of the amplitudes of modes 1 and 2 in simulations of the full model $(d)$, without fluid nonlinearity $(V=0)$ for $(a)$ and $(c)$, and without kinetic nonlinearity (no term in $\int f$ in equation (2)) for $(a)$ and $(b)$. The input parameters are given in table 2. Thin dotted curves indicated by arrows in $(b)$ : case with increased coupling coefficient, $V=400 \omega_{1}^{2} / Z_{0}$.

Let us now describe the evidence that supports the previous claims.

\subsection{Successive fluid then kinetic growth}

When, typically, $\nu_{f} \sim \nu_{d}$, subcritical instabilities can arise, even in the single-mode limit, if the initial amplitude is large enough [31]. In previous works [12-14], the kinetic subcritical instability was due to the growth of phase-space structures, and thus linked to chirping. In this case, we predicted that coupling to an unstable mode could provide the seed perturbation required for subcritical growth [15]. This is indeed what we observe with the present two-modes model.

Figure 2 shows a typical simulation in this regime. We observe a subcritical instability of mode 1 , and the amplitude grows to values much larger than the initial amplitude of mode 2. Meanwhile, as can be seen in the spectrogram $(b)$, the frequency of mode 1 chirps significantly. When $\delta \omega / \omega_{1} \approx 20 \%$, we stop the simulation, because an assumption of the model breaks down. Namely, in the simulation, the phase-shift $\theta$ is calculated by assuming that $\omega_{1}$ stays nearly constant.

Once fluid nonlinearity pushes the daughter amplitude to a large enough level, the subcritical instability is readily interpreted by the mechanism developed in [14]. Namely, the electric field of the daughter traps particles in a phase-space vortex, which is large enough to grow nonlinearly by climbing the positive velocity gradient of particle distribution. 

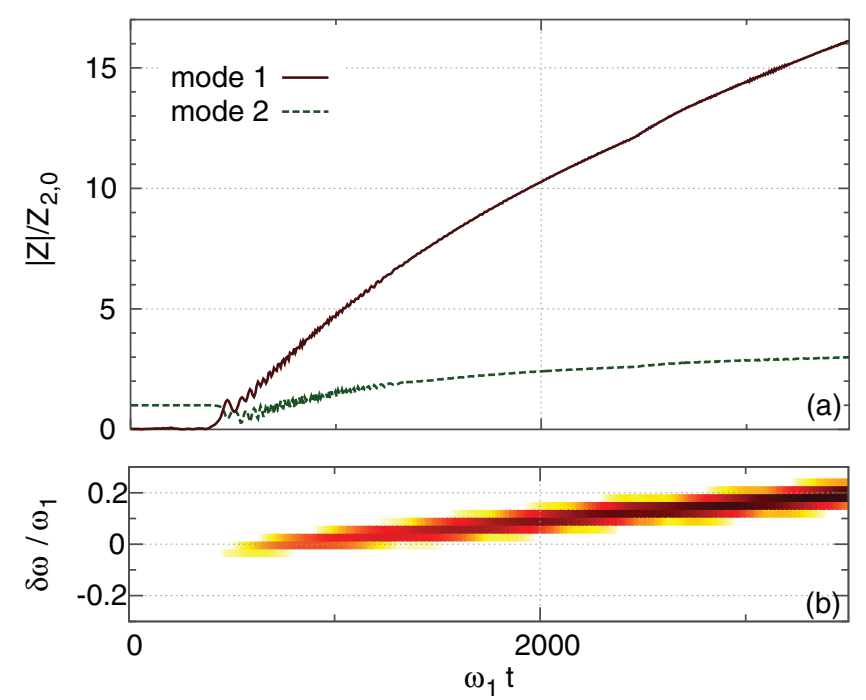

Figure 2. Time evolution of modes 1 and $2(a)$, and spectrogram of the total field $(b)$, in a simulation in the regime of successive fluid then kinetic growth. Input parameters are $\gamma_{L 0}=0.03 \omega_{1}, \gamma_{d}=0.031 \omega_{1}, \nu_{f}=1.3 \gamma_{L 0}, \nu_{d}=0.53 \gamma_{L 0}, V=60 \omega_{1}^{2} / Z_{0}$, $Z_{\text {noise }}=0.06 Z_{2,0}, Z_{2,0}=10^{-3} Z_{0}$, and $\mathrm{d} \omega_{2} / \mathrm{d} t=5 \times 10^{-4} \omega_{1}^{2}$ (same as figure 5 , except for an order-of-magnitude larger $\nu_{f}$ ).

This interpretation is further supported by the following numerical experiment. We switch off the fluid nonlinearity when the amplitude reaches a preset value. Figure 3 shows the time evolution of three simulations with the same input parameters, except for a different preset amplitude of switch-off. When the switch-off amplitude is larger than $\left|Z_{1}\right| / Z_{2,0} \approx 0.3$, the growth of mode 1 continues to much larger levels. This shows that the fluid nonlinearity is not necessary after an initial part of the growth phase. This, along with previous knowledge, indicates that fluid and kinetic nonlinearities can act in a successive manner to yield a subcritical instability.

\subsection{Collaborative fluid/kinetic nonlinearity}

When, typically, $\nu_{f} \ll \nu_{d}$, the single-mode model features no subcritical instability, even for large initial amplitude of perturbation [31]. In the two-modes case, significant subcritical instability requires a combination of fluid and kinetic nonlinearities. This new hybrid fluid-kinetic subcritical instability is illustrated in figure 4, which compares the stability of the daughter without $(a)$ and with $(b)$ the kinetic term in equation (2). In this figure, the stability of the daughter, for a fixed chirping rate, is represented in a two-dimensional parameter space $\left(\gamma_{d}-\gamma_{L 0}, V\right)$. Here, $\gamma_{d}-\gamma_{L 0}$ is used as a measure of distance from linear marginal stability. Figure 4 shows in color code the peak amplitude of the daughter mode, in this parameter space. Each point is the result of an ensemble average over 8 simulations with identical input parameters (the statistical variations are due to the random noise). We observe that the unstable region $\left(\max \left|Z_{1}\right| / Z_{2,0} \sim 1\right)$ is significantly extended to lower $V$ in the parameter space of $\left(\gamma_{d}-\gamma_{L 0}, V\right)$. In [32], a threshold condition has been derived analytically for the onset of abrupt daughter growth, as $Z_{2,0} V / \omega_{1}>\gamma_{d}$. It is shown by a series of circles in figure $4(b)$. There is a good

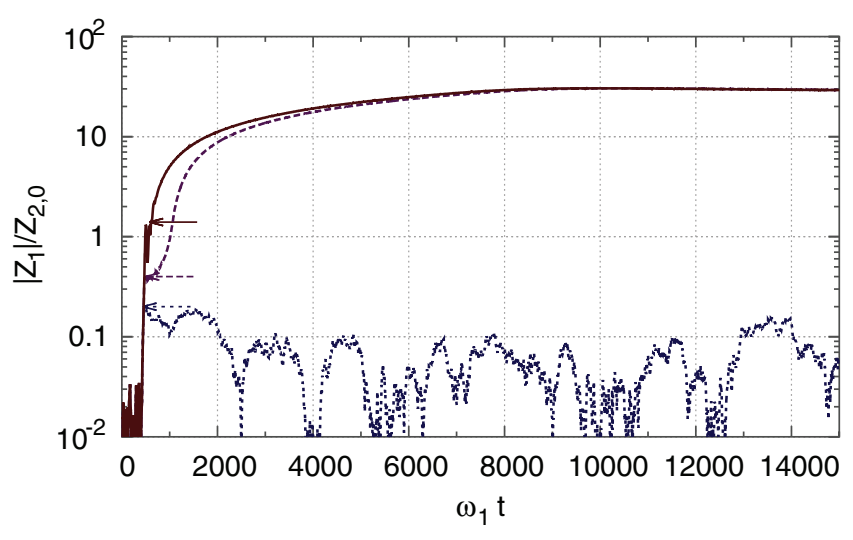

Figure 3. Time-evolution of the amplitude of mode 1 for the same parameters as figure 2, but where the fluid nonlinearity is switchedoff at a time marked by an arrow for each simulation.
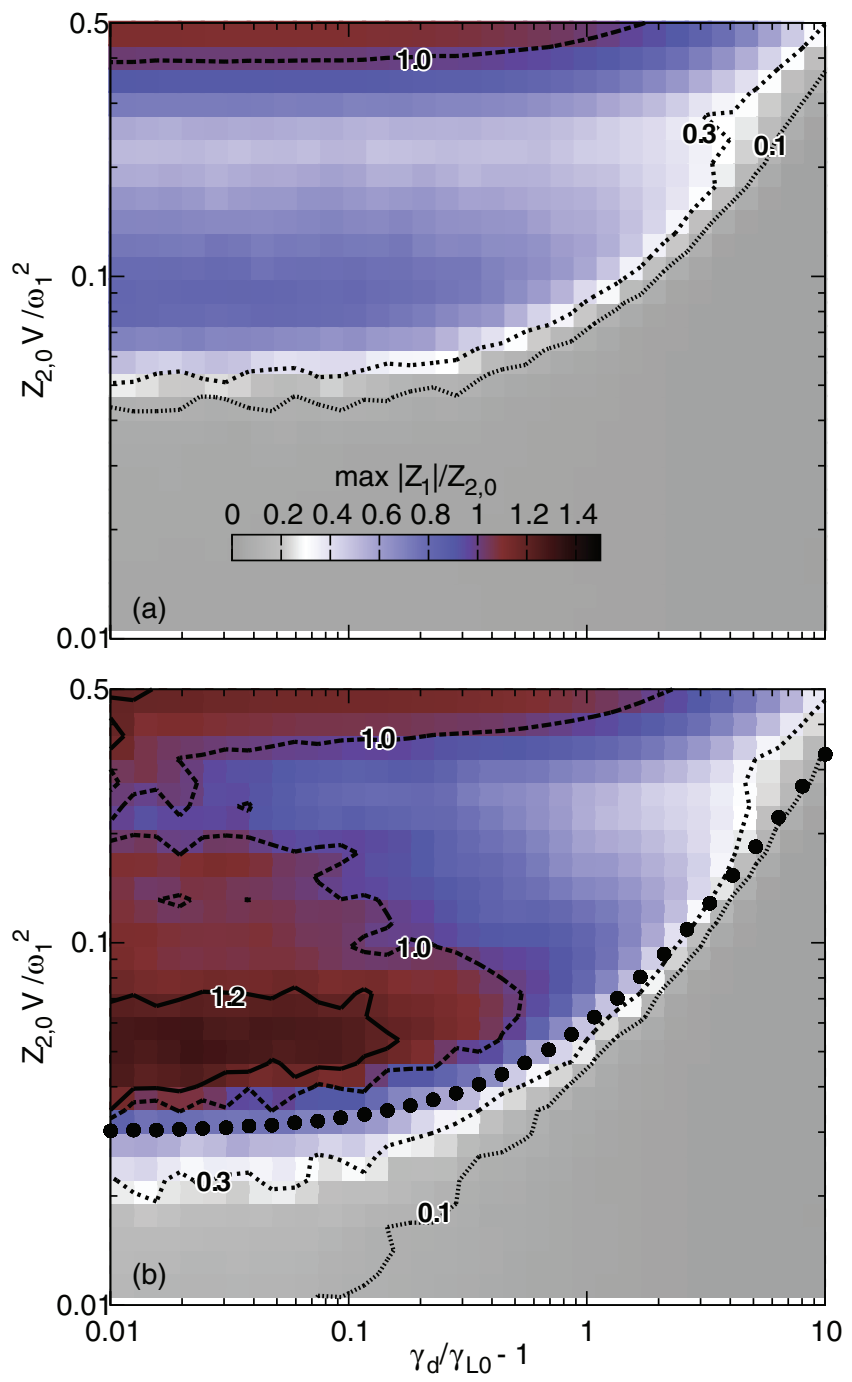

Figure 4. Nonlinear stability diagram for the daughter mode without $(a)$ and with $(b)$ the kinetic nonlinearity. Peak amplitude of the daughter mode as a function of both the distance from linear stability, and the coupling coefficient. The white area corresponds to the stability threshold in a sense, which will be explained at the end of section 5.1. Plain circles in $(b)$ show the threshold derived in [32]. 

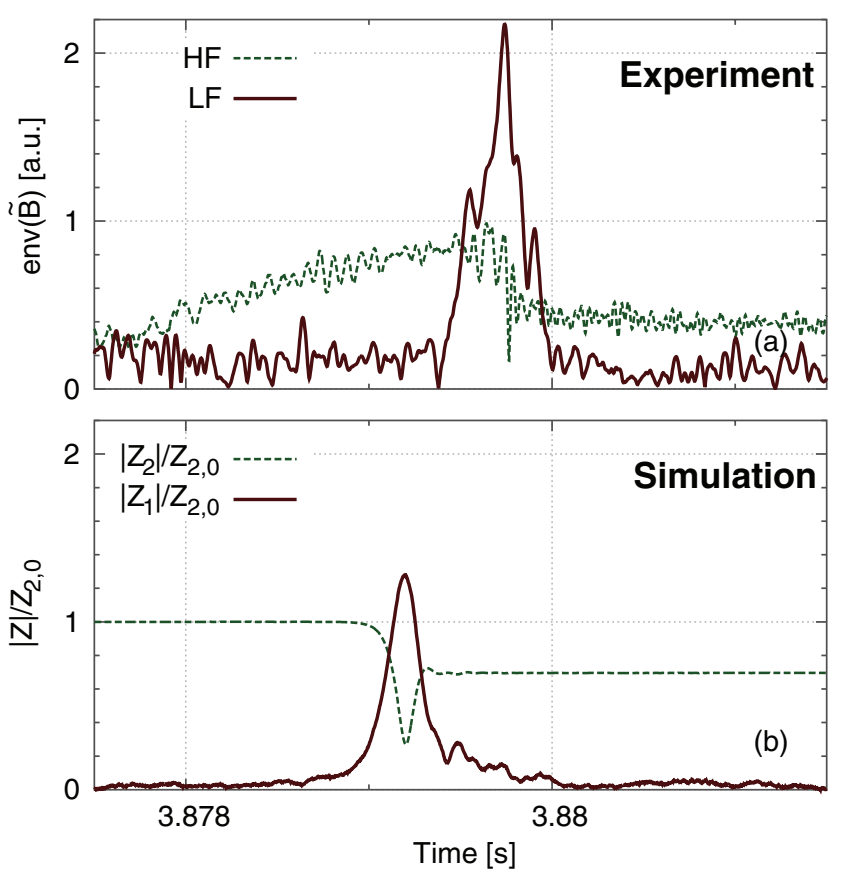

Figure 5. Comparison of the evolution of perturbation between the experiment and the simulation. (a) Time evolution of magnetic perturbation, filtered into low (LF) and high frequency (HF) components. Here, 'env' refers to the envelope. (b) Time evolution of the amplitudes of modes 1 and 2 in the simulation.

qualitative agreement with the stability threshold in the simulations, especially near linear marginality.

Figure $5(b)$ shows a typical simulation of the collaborative fluid/kinetic subcritical instability. We will describe in section 6 how this particular simulation is related to the LHD experiment shown in figure 5(a).

Figure 6 shows snapshots of the perturbed distribution function, at the time of maximum growthrate $(a)$, at the time of peak amplitude $(b)$, and at the time of maximum decay $(c)$ of the daughter mode. We observe that mode 1 is not significantly chirping during its growth, and only slightly chirping (by less than 10\%) during its decay. Indeed, the perturbation of particle distribution at the time of peak amplitude $(b)$ is centered around the resonant velocity, and apparently corresponds to a non-chirping BGK. We then observe accelerating holes and decelerating bumps, but only later, during the decay of daughter amplitude $(c)$. Therefore, the usual mechanism of kinetic subcritical growth, namely the acceleration of phasespace hole(s), is not responsible for the instability in this regime.

We can make a stronger statement, namely, that it is the sustained collaboration between fluid and kinetic nonlinearities that enables the subcritical growth in this regime. Indeed, in contrast with the results of figure 3 in the previous regime, $\left|Z_{1}\right|$ quickly decays back to noise level if we artificially disable the fluid nonlinearity at any point during the simulation.

Let us give more details about the mechanism of hybrid fluid-kinetic nonlinearity. It is convenient to describe the three terms in the rhs of equation (2) as kinetic term (with the integral), dissipative term (with $\gamma_{d}$ ), and coupling term (with $V$ ), respectively. The time evolution of the real part of these three terms is shown in figure $7(b)$, for a simulation with
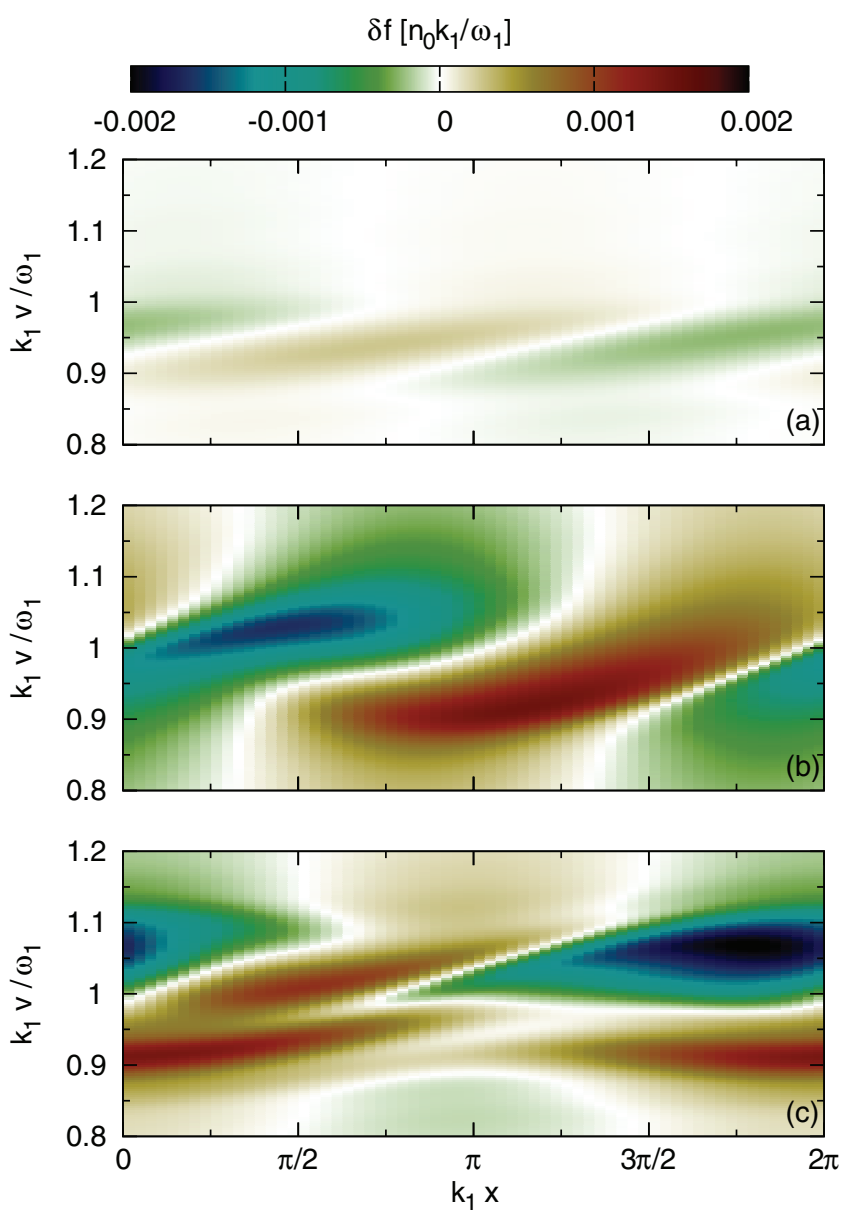

Figure 6. Snapshots of the perturbed particle distribution function in phase-space, at three consecutive times of the daughter mode's evolution: $(a)$ time of maximum growth-rate, $(b)$ time of peak amplitude, and $(c)$ time of maximum decay.

the same input parameters as the simulation in figure $5(b)$. During daughter growth, the dissipative and coupling terms are nearly locked in phase, with an opposite sign for the amplitude. We have indeed verified directly in our simulation that the phase difference between dissipation and coupling terms stays between $1.2 \pi$ and $1.3 \pi$ during the growth. Therefore, the coupling acts as an effective reduction of dissipation. The kinetic term is in phase with the dissipative term. In amplitude, all three terms are comparable, as shown in figure 7(c). Therefore, the sum of three terms approximately results in a real, positive growth rate $\sim \gamma_{d} \sim \gamma_{L 0}$. This can also be seen by defining an effective damping rate,

$$
\gamma_{d, \mathrm{eff}}=\gamma_{d}+\operatorname{Re}\left[\imath\left(V / \omega_{1}\right) Z_{2} Z_{1}^{*} Z_{1}^{-1} \mathrm{e}^{-\imath \theta t}\right] .
$$

The time evolution of $\gamma_{d \text {,eff }}$ is shown in figure $7(d)$. The main growth occurs when $\gamma_{d \text {,eff }}$ stays below $\gamma_{L 0}$.

\section{Simple limits}

In this section, let us consider two simpler limits. In the fluid limit, the kinetic nonlinearity (the term proportional to $\left.\int f \mathrm{e}^{-l\left(k_{1} x-\omega_{1} t\right)}\right)$ is neglected. In the kinetic limit, the fluid nonlinearity (the term proportional to $V Z_{2} Z_{1}^{*} \mathrm{e}^{-\imath \theta t}$ ) is neglected. 


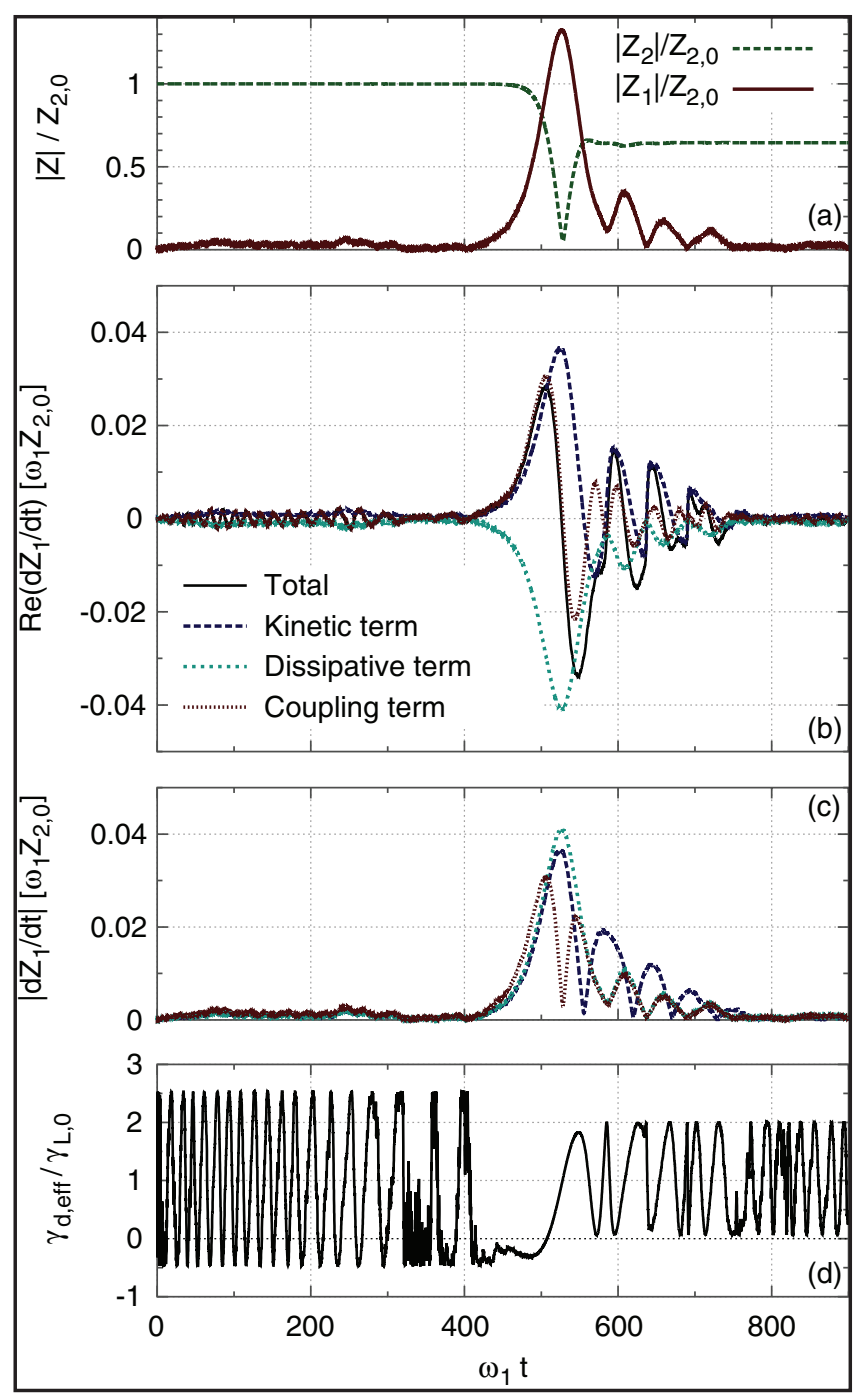

Figure 7. Collaboration of fluid and kinetic nonlinearities. (a) Time evolution of the amplitudes of the two modes. Time evolution of the real part $(b)$ and the absolute value $(c)$ of the three terms in the rhs of equation (2). $(d)$ Time evolution of the effective damping rate, $\gamma_{d, \mathrm{eff}}$, normalized to $\gamma_{L 0}$

\subsection{Fluid limit}

In the fluid limit, the integral in the rhs of equation (2) is dropped, and equation (1) can be ignored.

The theory in the dissipation-less $\left(\gamma_{d}=0\right)$ and chirpless $(\mathrm{d} \theta / \mathrm{d} t=0)$ limit, is very well understood. Here, we investigate the coupled evolution of two modes in the presence of both finite dissipation, and finite chirping. We choose $\gamma_{d}=0.031 \omega_{1}, \mathrm{~d} \theta / \mathrm{d} t=5 \times 10^{-4} \omega_{1}^{2}$, and noise level $Z_{\text {noise }} / Z_{2,0}=0.03$, because these values are used later in modeling the experiment. Figure $1(b)$ is an example of such a situation, with $V=50 \omega_{1}^{2} / Z_{2,0}$. Figure 8 shows the impact of the remaining free parameter, i.e. the coupling coefficient $V$. There are two striking features, which contrast with the dissipation-less chirp-less case.

- There is a sharp transition between stability $\left(Z_{1, \text { max }} \sim Z_{\text {noise }}\right)$ and instability $\left(Z_{1, \max } \sim Z_{2,0}\right)$.

- The relation between peak amplitude and coupling constant is non monotonous.

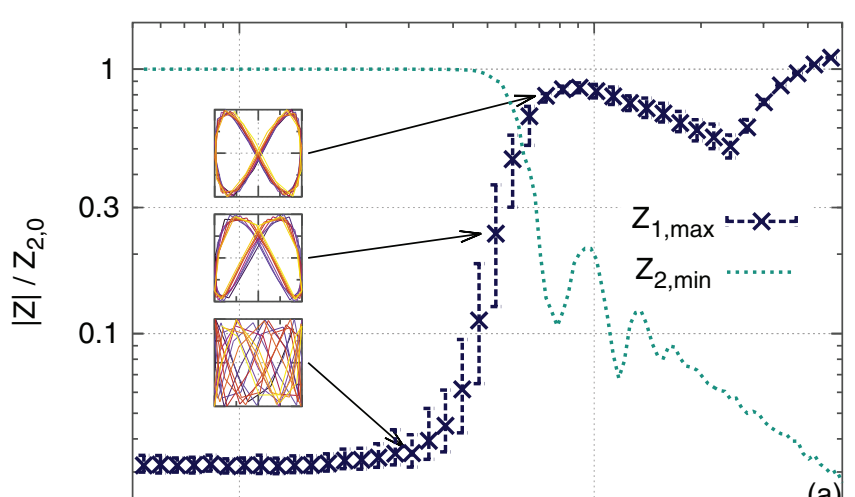

(a)

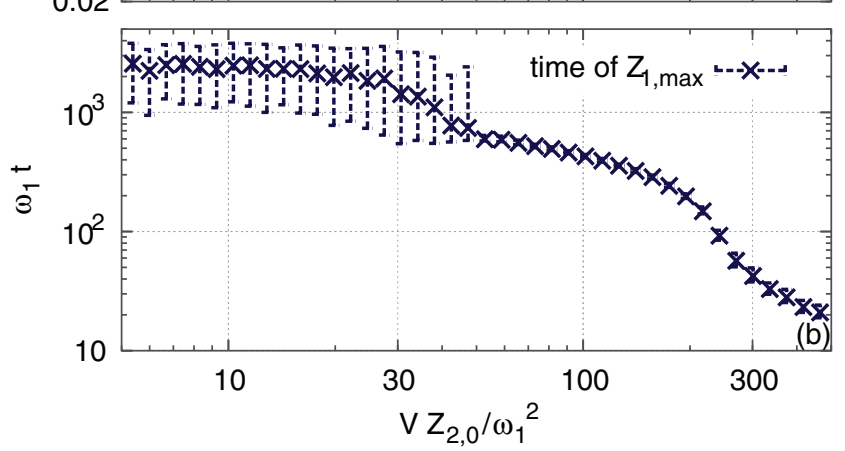

Figure 8. Impact of the coupling coefficient $V$ in the fluid limit. (a) Maximum amplitude of daughter mode, and minimum amplitude of mother mode. (b) Error bars correspond to the top of lower quartile, and bottom of upper quartile, of 128 ensembles. Insets: Lissajous diagrams for $\left(Z_{1}, Z_{2}\right)$, at $V=34,53$ and $73 \omega_{1}^{2} / Z_{2,0}$.

Figure $8(a)$ includes Lissajous diagrams $\left(\operatorname{Re} \tilde{Z}_{1} /\left|Z_{1}\right|\right.$ against Re $\tilde{Z}_{2} /\left|Z_{2}\right|$, where $\left.\tilde{Z}_{j} \equiv Z_{j} \mathrm{e}^{-l \omega_{j} t}\right)$ for three values of $V$, just below, at, and just above, the threshold value. Phase-locking occurs at and above the threshold. This suggests that the sharpness of the transition may be linked to a synchronization phenomenon.

The frequency ratio at the onset of daughter burst can be quite far from 2. For $V=53$, where phase-locking is observed, $\omega_{2} / \omega_{1}=1.77$ at the time of maximum growth. Therefore, note that phase-locking does not necessarily implies a ratio of 2 .

Note that for large values, $V>400 \omega_{1}^{2} / Z_{2,0}$, the daughter amplitude can become larger than the mother amplitude, without the help of the kinetic nonlinearity. However, in this case, the mother amplitude drops to $3 \%$ of its initial amplitude. This drop, and the timing with respect to the prescribed evolution of $\omega_{2}$ (see figure $1(b)$, dotted curves), are inconsistent with the experiment we interpret in section 6 .

Note that, since the peak amplitude is sensitive to $V$, one can loosely define the unstable region as a regime where the daughter reaches amplitudes comparable or much greater than the mother $\left(\max \left|Z_{1}\right| / Z_{2,0} \sim 1\right)$, and the stable region as the counterpart $\left(\max \left|Z_{1}\right| / Z_{2,0} \ll 1\right)$. In this sense, the white region in figure 4 , where $\max \left|Z_{1}\right| / Z_{2,0} \approx 0.3$, corresponds to the stability threshold.

\subsection{Single mode limit}

The system of equations (1) and (2), in the single mode $(V \rightarrow 0)$ limit, describes the subcritical excitation of an isolated 

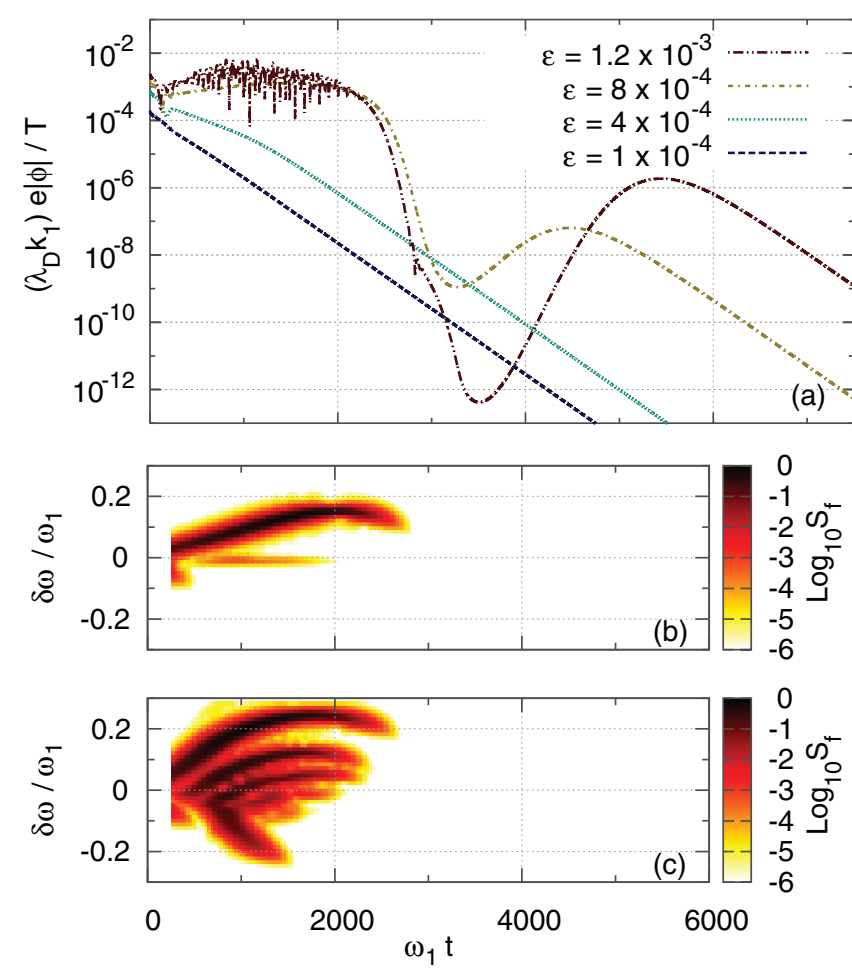

Figure 9. Time evolution $(a)$ and spectrograms $(b)$ and $(c)$ of electric potential for different initial amplitude of perturbation. The initial amplitudes in $(b)$ and $(c)$ are $\epsilon=8 \times 10^{-4}$ and $\epsilon=1.2 \times 10^{-3}$, respectively.

mode $E_{1}$ [12]. In this case, Landau damping generates a seed phase-space structure, whose growth rate can be positive if the growth due to momentum exchange overcomes decay due to collisions [14], which yields a threshold in initial, or noise amplitude.

Figure 9(a) shows time-series of electric field amplitude $\omega_{b}$ for different initial amplitudes. The input parameters are $\gamma_{d} / \gamma_{L 0}=1.05$ and $\nu_{f} / \gamma_{L 0}=\nu_{d} / \gamma_{L 0}=0.12$ which correspond to a linearly stable system. Fitting an exponentially decaying function to the simulation for small enough amplitude and at large enough $t$, we obtain the linear growth rate $\gamma_{0}=-0.045 \gamma_{L 0}$. Here, in the initial condition we apply a small perturbation, $f(x, v, t=0)=f_{0}(v)\left(1+\epsilon \cos k_{1} x\right)$, and the initial value of $Z_{1}$ is given by solving Poisson's equation.

However, for parameters relevant to typical instabilities in fusion plasmas, the threshold is much larger than thermal noise. Figure $1(c)$ is an example of such a situation. In fact, in the single mode $(V \rightarrow 0)$ limit, there is no subcritical instability unless we apply an artificially large initial perturbation or unless effective collisional drag is large enough, roughly $\nu_{f}>\nu_{d}$. This highlights the importance of the collaborative fluid/kinetic nonlinearity.

\section{Interpretation of LHD experiment}

Bursts of EGAMs with dynamical evolution of frequency (chirping) are routinely observed in tokamaks and stellerators. Suprisingly, as was reported in [18], an EGAM burst, with a $10 \mathrm{~ms}$ duration, is sometimes accompanied by a stronger burst, with a $1 \mathrm{~ms}$ duration, and up to twice the amplitude of the weaker burst. In [16], we have used the above model to analyze this observation. In this section, we provide more details on the latter analysis. We justify our assumption of fixed spatial profile of the daughter during its growth, based on experimental measurements. We discuss the role of the GAM continuum, and of the weak broadband signal, which is observed at the time of daughter growth. To allow quantitative analysis, we introduce scalar measures of the main properties of the daughter burst. These scalar measures are used to analyze the robustness of the modeling with respect to input parameters. Furthermore, observed correlations between these scalar measures provide readily-testable predictions.

\subsection{Modeling the experiment}

We concentrate on the LHD experiment, shot \#119729, at $t \approx 3.88 \mathrm{~s}$. The local plasma parameters around the radial location of mode $1\left(r_{1} \approx 0.06 \mathrm{~m}\right)$ are $B_{0} \approx 1.375 \mathrm{~T}, T_{\mathrm{i}} \approx 0.5-1 \mathrm{keV}$, $T_{\mathrm{e}} \approx 4 \mathrm{keV}, n_{e} \approx 10^{18} \mathrm{~m}^{-3}$, and $q \approx 2.5$. The ion species is hydrogen. The energetic particles originate from tangential NBI with $E_{b}=175 \mathrm{keV}$, and tangential major radius $R_{\mathrm{T}}=3.7 \mathrm{~m}$. The major radius of magnetic axis is $R_{0}=3.75 \mathrm{~m}$.

The density of energetic particles has not been measured in this experiment, but we may estimate the ratio between fast ions pressure $\beta_{\mathrm{h}}$ and thermal plasma pressure $\beta$ or thermal ion pressure $\beta_{i}$, to help situate the experimental conditions in terms of dimensionless parameters (although this is not used in any of the calculations in this paper). The density of energetic particles can be estimated [19] from the absorbed NBI power $P_{\mathrm{NBI}}=140 \mathrm{~kW}$ as $n_{\mathrm{NBI}} \approx 2 \times 10^{16} \mathrm{~m}^{-3}$, if we assume that the particle confinement time of the injected fast ions is $\approx 0.1 \mathrm{~s}$. Then, $\beta_{\mathrm{h}} / \beta \approx 0.7-0.8$, and $\beta_{\mathrm{h}} / \beta_{i} \approx 3.5-7$.

Figure 5(a) shows the time evolution of the magnetic perturbations. Here, the signal from the Mirnov coil has been filtered into a low frequency ( $\mathrm{LF}, f=30-50 \mathrm{kHz}$ ) component for the daughter mode, and a high frequency (HF, $f=60-95 \mathrm{kHz}$ ) component for the mother mode. From experimental measurements [18], the electric potential of the daughter mode is located in the core region, with a rather broad structure, $\Delta r / a \sim 0.5$, centered around $r / a \approx 0-0.1$. The spatial configuration agrees with this of a GAM. The toroidal mode number is $n=0$. The poloidal mode number is dominantly $m=0$ for the electric potential fluctuation, and $m=1$ for the density fluctuation (up-down anti-symmetric).

Since the spatial 3D structures of mother and daughter are very similar [18], we ignore the radial inhomogeneity, and study the ratio between mother and daughter amplitudes of magnetic perturbation. As measured by heavy-ion beam probe, the profile of electric potential (normalized by the peak amplitude) in the core is unchanged during the rapid growth of daughter mode in experiments. As for the outer region, the profile is inferred from the ratio between measured electric potential and amplitude of magnetic field perturbations. The latter ratio for the daughter is unchanged during the growth in experiments. These indicate that the spatial profile of the mode is nearly unchanged during the growth. This is consistent with our simple 1D model with constant input parameters. To relate the electric field in the simulation with the Mirnov coil signal, 
we assume a linear relationship between $|\tilde{\phi}|$ and $|\tilde{B}|$, which is consistent with experiment [19].

The dynamical change of frequency of the mother mode (mode 2), around the time of the burst of the daughter mode (mode 1), is modeled as a linear increase, with $\mathrm{d} \omega_{2} / \mathrm{d} t=5 \times 10^{-4} \omega_{1}^{2}$, where $\omega_{1}=2 \pi f_{1}$ and $f_{1}=43 \mathrm{kHz}$. Here, $f_{1}$ is obtained from reading the frequency of the peak in the spectrum of measured magnetic fluctuations at the time of maximum amplitude of the daughter.

We use four scalar measures to objectively characterize the evolution of the daughter:

(i) the maximum instantaneous growth rate $\gamma$,

(ii) the normalized peak amplitude, $A \equiv \max \left|Z_{1}\right| / Z_{2,0}$,

(iii) a scalar $\tau$ that measures the duration of daughter burst, and

(iv) the ratio $R^{\omega} \equiv \omega_{2}\left(t_{\text {growth }}\right) / \omega_{1}$ between mother and daughter frequencies at the time $t_{\text {growth }}$ of largest growth of the daughter.

Here, the duration $\tau$ of the daughter burst is defined as

$$
\tau \equiv \frac{1}{Z_{2,0}} \int\left|Z_{1}\right| S\left(\left|Z_{1}\right|\right) \mathrm{d} t
$$

where $S\left(\left|Z_{1}\right|\right)=1$ if $\left|Z_{1}\right|>Z_{\text {noise }}, 0$ else. For the experiment, we measure growth rate $\gamma=1.2 \times 10^{4} \mathrm{~s}^{-1}$, amplitude $A=2.17$, duration $\tau=0.6 \mathrm{~ms}$, and frequency ratio $R^{\omega}=1.9$. Note that $R^{\omega}$ is slightly below the perfect frequency matching condition $R^{\omega}=2$.

We scanned the parameter space $\left(\gamma_{L 0}, \gamma_{d}, \nu_{f}, \nu_{d}, V\right)$. We identified a finite region of the parameter space where $\gamma, A, \tau$ and $R^{\omega}$ in the simulation are in reasonable agreement with the experimental values. The experimental observation could also be reproduced by a wider range of input parameters $\left(Z_{\text {noise }}\right.$, $Z_{2,0}$, and $\left.\mathrm{d} \theta / \mathrm{d} t\right)$. Figure $5(b)$ shows the time evolution of the amplitudes $\left|Z_{1}\right|$ and $\left|Z_{2}\right|$ in the simulation. In Figure 5, time is scaled from normalized units to seconds by the coefficient $\omega_{1}$, and shifted in such a way that simulation and experiment are synchronized on the instant where $\omega_{2}(t) / \omega_{1}=1.5$, which we choose arbitrarily as the beginning of the simulation, as explained in section 2.3. For example, the beginning of the simulation here is at $t=3.8775 \mathrm{~s}$. Table 2 lists each input parameter (first and second columns), and its value used in the simulation of figure $5(b)$ (third column). In this simulation, we measure growthrate $\gamma=1.4 \times 10^{4} \mathrm{~s}^{-1}$, amplitude $A=1.34$, duration $\tau=0.6 \mathrm{~ms}$, and frequency ratio $R^{\omega}=1.8$. In addition to these four scalars, the simulation agrees qualitatively with the experiment in the sense that the daughter mode is only very slightly chirping $\left(\delta \omega / \omega_{1}<10 \%\right.$, as measured by tracking perturbations in the particle distribution), even though strongly chirping daughter mode is allowed in the model (self-consistently, albeit not consistently with physical assumptions). The lack of chirping of the daughter mode validates, a posteriori, our assumption of fixed $\omega_{1}$ in the frequency mismatch $\theta$ used for computing the wave-wave coupling terms.

Furthermore, the mother/daughter phase locking, which was discovered in [18], is qualitatively captured by numerical simulations, as shown in [16]. Therefore, we have shown that our model is able to qualitatively reproduce the nonlinear evolution of the daughter, in terms of amplitudes, timescales, and phase locking. We do not pretend to recover quantitatively from first principles the features of the daughter, neither to reproduce the combined evolution of both mother and daughter, but rather suggest the combined fluid-kinetic subcritical instability as a candidate mechanism for the strongest EGAM bursts in the LHD.

In our analysis, we have prescribed the time evolution of mother frequency $\omega_{2}(t)$ with constant chirping rate. As a caveat, this prescribed evolution ends when $\left|Z_{1}\right| \sim\left|Z_{2}\right|$. Indeed, in the experiment, the ratio $\omega_{2} / \omega_{1}$ increases very rapidly, but almost linearly, from 1.9 to 2.0 , within a $0.2 \mathrm{~ms}$ span during the daughter growth. The model, by its design, is unable to recover this apparent synchronization mechanism. However, the ratio of 2.0 is not reached before the very end of daughter growth. This indicates that the synchronization may not be a key aspect of the instability mechanism, although it may be a key aspect of the full dynamics of coupled modes. We can speculate that, although a ratio of 2.0 may be key in a quasi-stationary state, here the resonance condition may be broadened due the large growth rate of the daughter. Indeed, the daughter growth rate is $\gamma_{1} / \omega_{1} \sim 1 / 20$, therefore a ratio $\omega_{2} / \omega_{1} \sim 1.9$ (neglecting the broadening of the mother) could allow the resonance between mother and daughter. In other words, given the observed phase-locking during the growth phase, it appears that the rapid time evolution of $Z_{1}$ overcomes the finite frequency mismatch $\theta=\omega_{2}-2 \omega_{1}$ in $Z_{1}^{*} \mathrm{e}^{-\imath \theta t}$.

The role of the GAM continuum merits discussion. Unfortunately, there are large uncertainties in the measurements of $T_{\mathrm{i}}$ (measured by a neutral particle analyzer, integrated over a line of sight) and $T_{\mathrm{e}}$ (the uncertainty is of the order of $100 \%$ because of the very low density). Here $T_{\mathrm{e}}$ is important because $T_{\mathrm{e}} \gg T_{\mathrm{i}}$ in this experiment. Therefore, an accurate calculation of the GAM continuum, or even of the local GAM frequency, is left as an open issue. However, a rough estimation with rotational transform $\iota(r)=0.35+0.85(r / a)^{3}$, and temperature profiles peaking at $r=0$ below $1 \mathrm{keV}$ for $T_{\mathrm{i}}$ (measurements indicate a $0.5-1 \mathrm{keV}$ range), and around $4 \mathrm{keV}$ for $T_{\mathrm{e}}$, suggests that both daughter and mother may probably be above the GAM continuum.

In addition to the mother and the daugther, a weak, broad $(30-160 \mathrm{kHz})$ signal appears in the spectrogram at 3.879 55-3.879 $85 \mathrm{~s}$, which corresponds to the time of daughter growth. It appears to result from a parametric coupling of both mother and daughter with another mode, with a frequency in the range $10-15 \mathrm{kHz}$, which is present even before the mother is destabilized. Parametric coupling is speculated based on the relationship between frequencies of peaks, observed in the spectrum of $\tilde{B}$ at $t=3.8797 \mathrm{~s}$. By design, this additional physics is not captured by the present model. Its impact on mother and daughter may be negligible, since the amplitude of this mode is one order-of-magnitude below the amplitude of the daughter.

Reproducing the self-consistent coupled evolution of both mother and daughter is a relevant challenge that we leave for future work. This would require either solving a more advanced model, with two kinetic equations and two field 

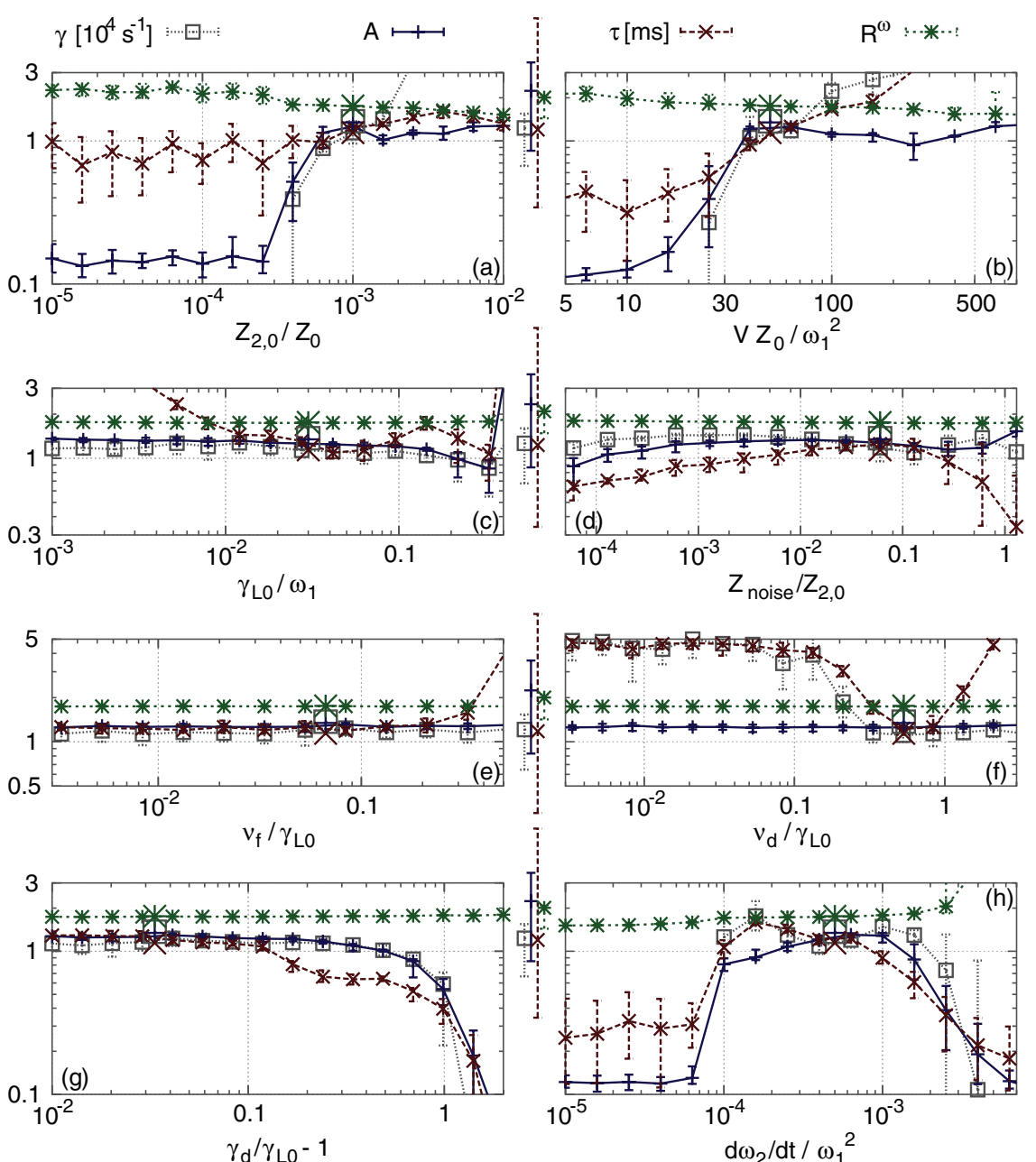

Figure 10. Impact of input parameters and free parameters of the model on the evolution of the daughter mode, in terms of the maximum instantaneous growth rate $\left(\gamma\left[10^{4} \mathrm{~s}^{-1}\right]\right)$, normalized peak amplitude $(A)$, duration of daughter burst $(\tau[\mathrm{ms}])$, and ratio of frequencies at the time of maximum growth $\left(R^{\omega}\right)$. The values for the selected simulation of figure 5 are shown by four larger symbols in each subplot. The values for the burst observed at $t \approx 3.88 \mathrm{~s}$ in the experiment are shown by points between the two columns of subplots, accompanied by vertical errorbars indicating the minimum and maximum value among the five daughter bursts observed in LHD shot \#119729. Impact of: initial amplitude of the mother mode $(a)$, coupling constant $(b)$, linear drive of the daughter mode $(c)$, noise level $(d)$, collisional drag $(e)$, collisional diffusion $(f)$, distance from linear marginality $(g)$, and chirping rate of the mother mode $(h)$.

equations, after obtaining the coupling term between $Z_{1}$ and $Z_{2}$ from 3D kinetic equations; or another approach altogether, such as full 3D gyrokinetic simulation. Here we focused on the instability mechanism of the daughter mode.

\subsection{Impact of input parameters}

The model includes a priori eight input parameters (assuming that the chirping rate $\mathrm{d} \theta / \mathrm{d} t$ for the mother mode around the onset of daughter is a constant). Here we describe the sensitivity, and the experimental and theoretical basis for these parameters.

We have conducted a sensitivity analysis, where we vary each input parameter, everything else being equal, and measure the impact on the time evolution of the daughter mode. Figure 10 shows the impact of each parameter. The fourth column (Range) of table 2 lists for each parameter the range (everything else being equal) where the simulation is in qualitative agreement with the experiment. Note that the evolution of the daughter is mostly sensitive to $\gamma_{L 0}, \nu_{d}, V, Z_{2,0}$ and $\mathrm{d} \omega_{2} / \mathrm{d} t$.
The fifth column of table 2 lists estimations from independent methods when available. Two of the five sensitive parameters, $Z_{2,0}, \mathrm{~d} \omega_{2} / \mathrm{d} t$, as well as $Z_{\text {noise }}$, are input from experimental data. Another sensitive parameter, $\nu_{d}$, as well as $\nu_{f}$, can be obtained from experimental measurements, by projecting the Fokker-Planck collision operator on the resonance surface of the daughter $[25,26]$, including the significant impact of impurities [23]. We use the local plasma parameters around the radial location of the daughter as given above, and a magnetic shear $S=0.2$. In addition, we assume carbon impurities with $T_{\mathrm{C}}=T_{\mathrm{i}}$ and $Z_{\text {eff }}=2$. We obtain $\nu_{f} / \gamma_{L 0} \approx 0.068$ and $\nu_{d} / \gamma_{L 0} \approx 0.44$, which are $1 \%$ and $17 \%$, respectively, below the parameters of the simulation shown in figure $5(b)$.

There remain two parameters with significant impacts: 1 . the slope of energetic particle distribution, parametrized by $\gamma_{L 0}$, and 2. the coupling coefficient $V$. For 1., it was shown that the order of magnitude $\gamma_{L 0} / \omega_{1} \sim 0.1$ is relevant for linearly unstable EGAMs on similar LHD plasmas [30], which suggests that 0.03 is relevant for linearly stable EGAMs. For 2., substituting the parameters of the experiment into 


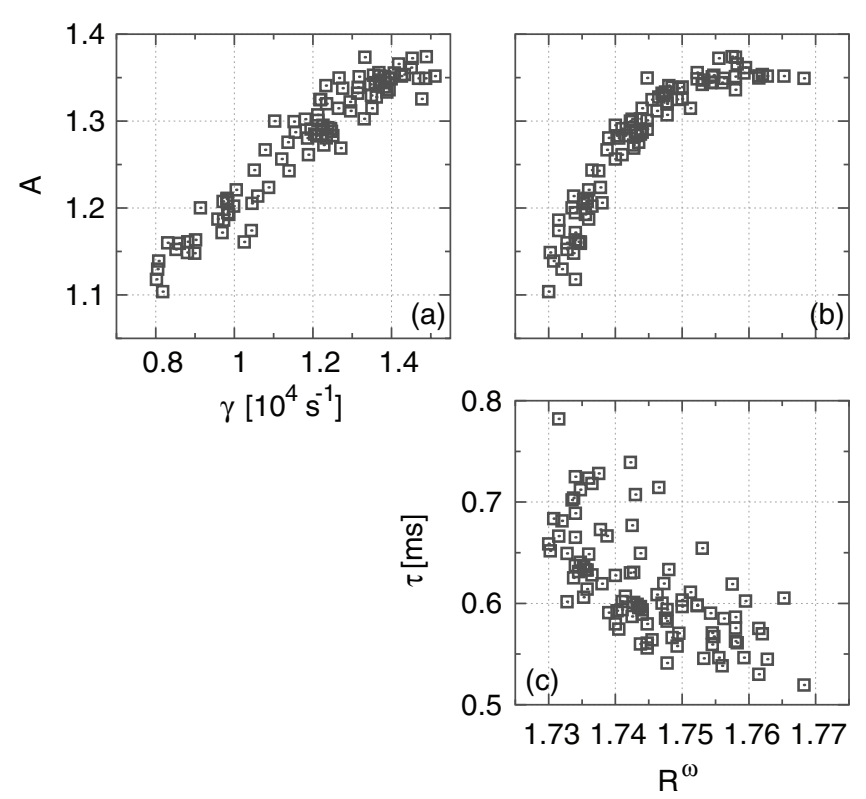

Figure 11. Correlations between scalar measures of daughter evolution. The parameters are the same as used in figure 5 .

equation (35) of [29] yields an estimate $Z_{0} V / \omega_{1}^{2} \sim 10^{-2}-10^{2}$ which is sensitive to the radial wave number of the GAM, but not inconsistent with our simulation. Thus, $V$ is a key parameter, with a finite range that reproduces the experiment, but with poor theoretical guide. Therefore, quantitative deduction of $V$ from first principles is encouraged.

\subsection{Predictions}

The model provides the following predictions, which are open to future experimental test.

1. The ratio between the mother and the daughter mode can become much larger, $\left|Z_{1}\right| /\left|Z_{2}\right| \gg 1$, and the daughter mode exhibit strong chirping, $\Delta \omega_{1} \sim \omega_{1}$, if the regime of successive fluid-kinetic subcritical instability is reached.

2. Since the best limit for driving a subcritical instability is $\mathrm{d} \omega_{2} / \mathrm{d} t \rightarrow 0$, and in this case, we observed no nonlinear instability for $\gamma_{d}>2 \gamma_{L 0}$, we predict that no subcritical instability will appear with $\gamma_{d} \gg \gamma_{L 0}$.

To make further predictions, it would be necessary to link the input parameters of the model with measurables such as equilibrium plasma profiles and energetic particle distribution. Unfortunately, an explicit connection would require us to derive the reduced model, including mode-mode coupling, from first principles, such as gyrokinetic equations. This is out of the scope of this paper.

Fortunately, it is possible to make other predictions, which can be straightforwardly tested in experiments, by looking for correlations, not between inputs and outputs as before, but between several outputs. For this, we take advantage of the variability of output, which originates from random noise. Figure 11 shows correlations between growth rate and peak amplitude $(a)$; between frequency ratio and peak amplitude $(b)$; and between frequency ratio and burst duration $(c)$. Each point corresponds to a simulation among an ensemble of 48 simulations, with identical input parameters (the same as those used in figure 5).

\section{Summary}

We have developed a reduced model for energetic particledriven, nonlinear excitation of subcritical instabilities in toroidal plasma. The model combines a 1D kinetic equation with equations for period doubling. The kinetic equation approximately describes wave-particle interactions between fast ions and a single MHD mode in a toroidal plasma, such as an EGAM, or a toroidal Alfvén eigenmode.

Two regimes have been investigated. In a first regime, of successive fluid then kinetic growth, the dormant subcritical mode is first triggered by fluid coupling to the supercritical mode, which allows it to reach amplitudes of the same order of magnitude as the supercritical mode. This amplitude is above the threshold for the conventional kinetic subcritical instability $[12,14]$. Then, the amplitude can keep growing by momentum exchange between the wave and phase-space structure(s), accompanied by significant chirping. In a second regime, of collaborative fluid-kinetic growth, the subcritical growth is due to an uninterrupted collaboration between fluid and kinetic nonlinearities. This is a new kind of instability mechanism, where fluid and kinetic nonlinearities have similar (in amplitude) contributions to the mode growth. Contrarily to the mechanism developed in earlier theories [12,14], the growth occurs much below the amplitude threshold, and without chirping. Typically, the ratio $\nu_{f} / \nu_{d}$ selects one or the other regime. The first regime is obtained for $\nu_{f} / \nu_{d} \sim 1$ and above. In this case, the amplitude of the subcritical mode can grow orders-of-magnitude above the amplitude of the supercritical mode.

We have shown that the model can reproduce key aspects of the experimental observation of [18]. It interprets the daughter mode as a manifestation of the collaborative fluid-kinetic subcritical instability. In contrast with previously-known kinetic subcritical instabilities, the amplitude stays below the kinetic threshold, and chirping seems to be limited by a quasi-phasematching condition with the mother mode. These results imply a new channel of mode excitation, which modifies the flow of energy in the system.

\section{Acknowledgments}

One of the authors (ML) is grateful for stimulating discussions with M. Yagi, H. Wang, M. Kikuchi, K. Miki, and the participants in the Festival de Théorie. This work was supported by grants-in-aid for scientific research of JSPS, Japan (15K18305, 15H02155, 23244113 and 15H02335), by the collaboration programs of the RIAM of Kyushu University and of NIFS, and Asada Science Foundation, and by the EUROfusion consortium and the French Research Federation for Fusion Studies. The views and opinions expressed herein do not necessarily reflect those of the European Commission. Computations were performed on the XT system at Kyushu University and Plasma Simulator at NIFS. 


\section{Appendix}

It is possible to approximate the problem of wave-particle interactions between an EGAM and fast ions by a simple harmonic oscillator. This requires expansion of the perturbed Hamiltonian around a resonant phase-space surface. This reduction from $3 \mathrm{D}$ to $1 \mathrm{D}$ is developed here for tokamak geometry.

For far-passing particles, the resonance condition is $\Omega=\omega_{\text {EGAM }}$, where

$$
\Omega=l \omega_{\theta},
$$

where $\omega_{\theta}=v_{\|} / q R_{0}$ is the frequency of poloidal motion, and $l$ is a non-zero integer. If we assume that resonant interactions are dominated by a population corresponding $l=1$, we can simplify following discussions.

The gyrokinetic equation, can be put in Hamiltonian form,

$$
\frac{\partial f}{\partial t}-\{H, f\}=\left.\frac{\mathrm{d} f}{\mathrm{~d} t}\right|_{\text {coll. }},
$$

where $H$ is the Hamiltonian, and \{\} are Poisson brackets. In [33], the Hamiltonian is obtained in action-angle variables, $(\boldsymbol{\alpha}$, $\boldsymbol{J})$, where $\boldsymbol{\alpha}=(\xi, \zeta, \theta)$, and $\boldsymbol{J}=\left(J_{\xi}, J_{\zeta}, J_{\theta}\right)$, in arbitrary tokamak geometry. The canonical angles $\theta$ and $\zeta$ reduce to the geometric poloidal and toroidal angles if we neglect finite aspect ratio effects. The canonical angle $\xi$ corresponds to the gyrokinetic angle. The unperturbed part of the Hamiltonian, $H_{0}(\boldsymbol{J})$, which is integrable, yields the $3 \mathrm{D}$, unperturbed particle trajectories.

The electric potential perturbation of an EGAM is dominated by a zonal $(n=0, m=0)$ component $\phi_{0}$, with coupling to $n=0, m=1,2, \ldots$ components [34], $\phi_{1}, \phi_{2} \ldots$ The amplitude of $\phi_{m+1}$, relative to the $\phi_{m}$, is of the order of $k_{r} \rho_{i} T_{\mathrm{e}} / T_{\mathrm{i}}$, which may be of the order of $10^{-1}$ in the LHD experiment where $T_{\mathrm{e}} / T_{\mathrm{i}} \sim 10$ (the radial wave number $k_{r}$ is not measured, but typically, $k_{r} \rho_{i} \sim \rho_{i} / a \sim 10^{-2}$ for an EGAM). The zonal component cannot extract free energy from the energy gradient of energetic particle population (this can be seen from the linear gyrokinetic equation in, e.g. [35]). Therefore, to model resonant wave-particle interactions, $\phi_{1}$ plays a crucial role, as the lowest order relevant component. It is the perturbation to particle trajectories induced by $\phi_{1}$ that we aim to model, on a timescale much shorter than the timescale of evolution of $H_{0}$. In other words, we assume fixed 3D plasma equilibrium, 3D eigenmode spatial structure, and 3D unperturbed orbits.

The $n=0, m=1$ component of the EGAM can be described by a perturbation to the Hamiltonian,

$$
H_{1}=e \phi_{1} \text {. }
$$

Writing $\phi_{1}=\hat{\phi}_{1} \exp \imath\left(\theta+k_{r}\left(r-r_{0}\right)-\omega t\right)+$ c.c., yields

$$
H_{1}=C(\boldsymbol{J}) \mathrm{e}^{l(\theta-\omega t)}+\text { c.c., }
$$

with $C=e \hat{\phi}_{1} \exp \imath\left[k_{r}\left(r-r_{0}\right)\right]$.

The resonance condition, $\omega=\omega_{\theta}(\boldsymbol{J})$, where $\omega_{\theta} \equiv \frac{\partial H_{0}}{\partial J_{\theta}}$, is satisfied on a resonant phase-space surface. Formally, the resonant phase-space surface, $\boldsymbol{J}=\left\{\boldsymbol{J}^{R}\right.$ such that $\left.J_{\theta}^{R}=F\left(J_{\xi}^{R}, J_{\zeta}^{R}\right)\right\}$, is defined by a function $F$.

Once the perturbed Hamiltonian has been put in the form of equation (A.4), we can reduce the problem to one action and one angle [36, 37], by performing a canonical transformation $\boldsymbol{J} \cdot \mathrm{d} \boldsymbol{\alpha}-H \mathrm{~d} t=\boldsymbol{I} \cdot \mathrm{d} \boldsymbol{\psi}-H^{\prime} \mathrm{d} t+\mathrm{d} S$ with the generating function

$$
\begin{aligned}
S= & -\boldsymbol{I} \cdot \boldsymbol{\psi}+I_{\theta}(\theta-\omega t) \\
& +I_{\xi} \xi+I_{\zeta} \zeta+F\left(I_{\xi}, I_{\zeta}\right) \theta
\end{aligned}
$$

This procedure yields the new actions $I_{\xi}=J_{\xi}, \quad I_{\zeta}=J_{\zeta}$, $I_{\theta}=J_{\theta}-F\left(I_{\xi}, I_{\zeta}\right)$, new angles $\psi_{\xi}=\xi+\theta \partial F / \partial I_{\xi}, \psi_{\zeta}=\zeta+$ $\theta \partial F / \partial I_{\zeta}, \psi_{\theta}=\theta-\omega t$, and $H=H^{\prime}+\omega I_{\theta}$. Thus, near the resonant phase-space surface, $J_{\theta}=J_{\theta}^{R}+I_{\theta}$, and we can expand the new Hamiltonian around this surface,

$$
\begin{aligned}
H^{\prime}(\boldsymbol{\psi}, \boldsymbol{I})= & H_{0}\left(J_{\xi}^{R}, J_{\zeta}^{R}, J_{\theta}^{R}+I_{\theta}\right) \\
& -I_{\theta} \omega+C(\boldsymbol{J}) \mathrm{e}^{\imath(\theta-\omega t)}+\text { c.c. } \\
= & H_{0}\left(\boldsymbol{J}^{R}\right)+I_{\theta}\left(\omega_{\theta}\left(\boldsymbol{J}^{R}\right)-\omega\right) \\
& +\frac{1}{2} D I_{\theta}^{2}+C(\boldsymbol{J}) \mathrm{e}^{\iota \psi_{\theta}}+\text { c.c. }
\end{aligned}
$$

with $D\left(\boldsymbol{J}^{R}\right) \equiv \partial^{2} H_{0} / \partial J_{\theta}^{2}\left(\boldsymbol{J}^{R}\right)=\partial \omega_{\theta} / \partial J_{\theta} \sim 1 /\left(m_{i} q^{2} R_{0}^{2}\right)$, where $m_{i}$ is the mass of resonating ions.

If the variations of $H(\boldsymbol{J})$ are small around $\boldsymbol{J}^{R}$, we can replace $C(\boldsymbol{J})$ by $C\left(J^{R}\right)$ in the latter expression. Assuming, further, that $\phi_{1}$ peaks at the resonant surface, $C\left(\boldsymbol{J}^{R}\right)=e \hat{\phi}_{1}$. Then, we obtain the new Hamiltonian $H^{\prime}=H_{0}\left(\boldsymbol{J}^{R}\right)+H_{1, J^{R}}\left(\psi_{\theta}, I_{\theta}\right)$, with

$$
H_{1, J^{R}}(\psi, I) \equiv \frac{1}{2} D I^{2}+e \hat{\phi}_{1} \mathrm{e}^{\imath \psi}+\text { c.c.. }
$$

Thus, the problem has been reduced to a 1D Hamiltonian problem for the angle-action variables $(\psi, I) \equiv\left(\psi_{\theta}, I_{\theta}\right)$. The new angle is $\psi=\theta-\omega t$, and the new action is $I=J_{\theta}-J_{\theta}^{R}$. For passing particles, and to the zeroth order in $\rho^{*}$,

$$
J_{\theta}=e \phi_{\mathrm{T}}+\oint \frac{m_{i} v_{\|} B}{2 \pi \boldsymbol{B} \cdot \nabla \theta} \mathrm{d} \theta
$$

where $\phi_{\mathrm{T}}$ is the toroidal magnetic flux normalized by $2 \pi$. Therefore, $I$ is roughly proportional to $\sim v_{\|}-v_{\|}^{R}$. We have shown that, in the neighborhood of the resonant surface, $H_{1}$, which yields the EGAM-induced perturbation to unperturbed $3 \mathrm{D}$ orbits, is essentially $1 \mathrm{D}$.

On the other hand, the Hamiltonian for a single sine wave $(k, \omega)$ in a 1D plasma, expressed in a frame moving at the wave phase velocity $v^{R}=\omega / k$, can also be expressed in angleaction variables,

$$
h=\frac{k^{2}}{2 m_{i}} I_{1 \mathrm{D}}^{2}+e \hat{\phi} \mathrm{e}^{i \psi_{1 \mathrm{D}}}+\text { c.c. },
$$

where $\psi_{1 \mathrm{D}} \equiv k x-\omega t$ and $I_{1 \mathrm{D}} \equiv m_{i}\left(v-v^{R}\right) / k$. The form of the Hamiltonian is shared with the Harmonic oscillator, as well as the approximate Hamiltonian of the EGAM, equation (A.8). In this sense, the model of resonant interactions between fast ions and a single electrostatic wave in a 1D plasma, is isomorphic to a whole class of reduced models of interactions between fast ions and a single MHD mode in a toroidal plasma, such as an EGAM, or a toroidal Alfvén eigenmode as well [22]. 


\section{References}

[1] Yoshizawa A., Itoh S. and Itoh K. 2002 Plasma and Fluid Turbulence: Theory and Modelling (Series in Plasma Physics and Fluid Dynamics) (Boca Raton, FL: CRC Press)

[2] Romanov V. 1973 Funct. Anal. Appl. 7 137-46

[3] Yagi M., Itoh S., Itoh K., Fukuyama A. and Azumi M. 1995 Phys. Plasmas 2 4140-8

[4] Drake J.F., Zeiler A. and Biskamp D. 1995 Phys. Rev. Lett. 75 4222-5

[5] Weissman M.A. 1979 Phil. Trans. R. Soc. A $290639-81$

[6] Meignin L., Gondret P., Ruyer-Quil C. and Rabaud M. 2003 Phys. Rev. Lett. 90234502

[7] Carrera R., Hazeltine R.D. and Kotschenreuther M. 1986 Phys. Fluids 29 899-902

[8] Eliasson B. and Shukla P. 2006 Phys. Rep. 422 225-90

[9] Schamel H. 1979 Phys. Scr. 20336

[10] Dupree T.H. 1982 Phys. Fluids 25 277-89

[11] Schamel H. 2012 Phys. Plasmas 19020501 (pages 17)

[12] Berk H.L., Breizman B.N., Candy J., Pekker M. and Petviashvili N.V. 1999 Phys. Plasmas 6 3102-13

[13] Lesur M., Idomura Y. and Garbet X. 2009 Phys. Plasmas 16092305

[14] Lesur M. and Diamond P.H. 2013 Phys. Rev. E 87031101

[15] Lesur M., Diamond P.H. and Kosuga Y. 2014 Plasma Phys. Control. Fusion 56075005

[16] Lesur M., Itoh K., Ido T., Osakabe M., Ogawa K., Shimizu A., Sasaki M., Ida K., Inagaki S., Itoh S.I. and the LHD experiment group 2016 Phys. Rev. Lett. 116015003

[17] Berk H.L., Breizman B.N. and Pekker M. 1995 Phys. Plasmas 2 3007-16

[18] Ido T., Osakabe M., Shimizu A., Ogawa K., Nishiura M., Lesur M., Itoh K., Ida K., Inagaki S. and Itoh S.I. 2016 Phys. Rev. Lett. 116015002

[19] Ido T. and Group T.L.E. et al 2015 Nucl. Fusion 55083024

[20] Manley J. and Rowe H. 1956 Proc. IRE 44 904-13
[21] Breizman B.N., Berk H.L., Pekker M.S., Porcelli F., Stupakov G.V. and Wong K.L. 1997 Phys. Plasmas 4 1559-68

[22] Lesur M. 2011 The Berk-Breizman model as a paradigm for energetic particle-driven Alfvén eigenmodes PhD Thesis Ecole Polytechnique, France

[23] Lesur M., Idomura Y., Shinohara K., Garbet X. and the JT-60 Team 2010 Phys. Plasmas 17122311 (page 9)

[24] Helander P. and Sigmar D. 2002 Collisional Transport in Magnetized Plasmas (Cambridge: Cambridge University Press)

[25] Berk H.L., Breizman B.N. and Pekker M.S. 1997 Nonlinear theory of kinetic instabilities near threshold NTIS Report DOE/ER/54346-787

[26] Lilley M.K., Breizman B.N. and Sharapov S.E. 2009 Phys. Rev. Lett. 102195003

[27] Sasaki M., Itoh K., Nagashima Y., Ejiri A. and Takase Y. 2009 Phys. Plasmas 16022306

[28] Hallatschek K. and Biskamp D. 2001 Phys. Rev. Lett. 86 1223-6

[29] Itoh K., Hallatschek K., Itoh S.I., Diamond P.H. and Toda S. 2005 Phys. Plasmas 12062303

[30] Wang H. and Todo Y. 2013 Phys. Plasmas 20012506

[31] Lesur M. and Idomura Y. 2012 Nucl. Fusion 52094004

[32] Itoh K., Itoh S.I., Kosuga Y., Lesur M. and Ido T. 2016 Plasma Phys. Rep. at press

[33] Berk H.L., Breizman B.N. and Pekker M.S. 1995 Nucl. Fusion 35 1713-20

[34] Sasaki M., Itoh K., Ejiri A. and Takase Y. 2008 Plasma Fusion Res. 3009

[35] Qiu Z., Zonca F. and Chen L. 2010 Plasma Phys. Control. Fusion 52095003

[36] Lichtenberg A. 1969 Phase-Space Dynamics of Particles (Wiley Series in Plasma Physics) (New York: Wiley)

[37] Garbet X., Dif-Pradalier G., Nguyen C., Angelino P., Sarazin Y., Grandgirard V., Ghendrih P. and Samain A. 2008 AIP Conf. Proc. 1069 271-6 